\title{
Addressing widespread misidentifications of traditional medicinal mushrooms in Sanghuangporus (Basidiomycota) through ITS barcoding and designation of reference sequences
}

\author{
Shan Shen ${ }^{1,2+}$, Shi-Liang Liư ${ }^{1+}$, Ji-Hang Jiang ${ }^{1}$ and Li-Wei Zhou ${ }^{1,3^{*}}$ (D)
}

\begin{abstract}
"Sanghuang" refers to a group of important traditionally-used medicinal mushrooms belonging to the genus Sanghuangporus. In practice, species of Sanghuangporus referred to in medicinal studies and industry are now differentiated mainly by a BLAST search of GenBank with the ITS barcoding region as a query. However, inappropriately labeled ITS sequences of "Sanghuang" in GenBank restrict accurate species identification and, to some extent, the utilization of these species as medicinal resources. We examined all available 271 ITS sequences related to "Sanghuang" in GenBank including 31 newly submitted sequences from this study. Of these sequences, more than half were mislabeled so we have now corrected the corresponding species names. The mislabeled sequences mainly came from strains utilized by non-taxonomists. Based on the analyses of ITS sequences submitted by taxonomists as well as morphological characters, we separate the newly described Sanghuangporus subbaumii from S. baumii and treat $S$. toxicodendri as a later synonym of S. quercicola. Fourteen species of Sanghuangporus are accepted, with intraspecific distances up to $1.30 \%$ (except in S. vaninii, S. weirianus and S. zonatus) and interspecific distances above 1.30\% (except between S. alpinus and S. Ionicerinus, and S. baumii and S. subbaumii). To stabilize the concept of these 14 species of Sanghuangporus, their taxonomic information and reliable ITS reference sequences are provided. Moreover, ten potential diagnostic sequences are provided for Hyperbranched Rolling Circle Amplification to rapidly confirm three common commercial species, viz. S. baumii, S. sanghuang, and S. vaninii. Our results provide a practical method for ITS barcoding-based species identification of Sanghuangporus and will promote medicinal studies and commercial development from taxonomically correct material.
\end{abstract}

Keywords: Hymenochaetaceae, Phylogeny, Species boundary, Taxonomy, Wood-inhabiting fungi, One new taxon

* Correspondence: liwei_zhou1982@im.ac.cn

†'Shan Shen and Shi-Liang Liu contributed equally to this work.

'State Key Laboratory of Mycology, Institute of Microbiology, Chinese

Academy of Sciences, Beijing 100101, China

${ }^{3}$ Institute of Applied Ecology, Chinese Academy of Sciences, Shenyang

110016, China

Full list of author information is available at the end of the article

(c) The Author(s). 2021 Open Access This article is licensed under a Creative Commons Attribution 4.0 International License, which permits use, sharing, adaptation, distribution and reproduction in any medium or format, as long as you give appropriate credit to the original author(s) and the source, provide a link to the Creative Commons licence, and indicate if changes were made. The images or other third party material in this article are included in the article's Creative Commons licence, unless indicated otherwise in a credit line to the material. If material is not included in the article's Creative Commons licence and your intended use is not permitted by statutory regulation or exceeds the permitted use, you will need to obtain permission directly from the copyright holder. To view a copy of this licence, visit http://creativecommons.org/licenses/by/4.0/ 


\section{INTRODUCTION}

Many macrofungi are established in traditional medicine and possess diverse properties (Wu et al. 2019a). "Sanghuang" comprises an important group of woodinhabiting mushrooms that have been utilized in traditional medicine in China and adjacent countries for 2000 years (Zhou et al. 2020). Modern scientific studies have revealed several medicinal attributes of "Sanghuang", including antitumor, antioxidant, anti-inflammation, and immunomodulation activities (Zhou et al. 2020). This fungal resource has also attracted the attentions of fungal chemists and pharmacologists outside Asia (Chepkirui et al. 2018; Cheng et al. 2019). Natural products, such as polysaccharides, polyphenols, pyrones and terpenes are the bioactive compounds responsible for the medicinal properties of "Sanghuang" (Zhou et al. 2020). Today, "Sanghuang" is mainly consumed in a brewed tea made from small pieces of cultivated basidiomes or occasionally powdered mycelia.

Like other wood-inhabiting traditional medicinal mushrooms, such as "Lingzhi" (Cao et al. 2012; Wang et al. 2012; Yao et al. 2013, 2020; Dai et al. 2017), "Niuchangchih" (Wu et al. 2012b, 2012c) and "Fuhling" (Redhead and Ginns 2006), there has been much debate about the taxonomic identity of "Sanghuang". Most fungal taxonomists now agree that "Sanghuang" is represented by species of Sanghuangporus (Zhou et al. 2020). Fourteen species have been described and accepted as members of Sanghuangporus: 11 species in Asia, and one in each of Africa, Europe, and North America (Zhou et al. 2020). In addition, more new species await to be described from Africa (Chepkirui et al. 2018; Cheng et al. 2019) and perhaps other parts of the world. Besides morphological and ecological (host preference) characters, the ITS barcoding region provides the most powerful tool for differentiating species of the genus. For example, more than half of the known species of Sanghuangporus were discovered with the aid of the ITS region alone (Wu et al. 2012a, 2019b; Tian et al. 2013; Ghobad-Nejhad 2015; Tomšovský 2015; Zhu et al. 2017). Moreover, the reliability of the ITS region for species differentiation in the genus has been substantiated by a multilocus-based phylogenetic analysis (Zhu et al. 2019). Consequently, Zhou et al. (2020) reported ITS sequences from reliably identified voucher collections of the known species in the genus.

Transdisciplinary studies on Sanghuangporus have been performed to promote the utilization of this medicinal resource (Zhou et al. 2016; Cai et al. 2019; Zhu et al. 2019; Shao et al. 2020). Most of these studies aimed to identify their materials via a BLAST search of GenBank (https://www.ncbi.nlm.nih.gov/genbank/) using the ITS barcoding region as the query. However, even though each of the 14 species of Sanghuangporus has a reliable ITS sequence accession number (Zhou et al.
2020), it is not always easy to determine material in hand by a simple ITS-based BLAST search. This is a consequence of redundant and even incorrectly labeled ITS sequences in GenBank (Nilsson et al. 2006; Hofstetter et al. 2019). With inaccurately identified sequences emerging as potential matches, more collections will inevitably be inaccurately identified and the ITS sequences generated from the inaccurately identified collections will be submitted to GenBank compounding the issue and presenting new obstacles for later accurate identification. This means that there is high likelihood of medicinal and other attributes being attributed to incorrectly named species of "Sanghuang". Meanwhile, before the erection of the genus Sanghuangporus (Zhou et al. 2016), ITS sequences generated from "Sanghuang" were labeled under other generic names, such as Inonotus and Phellinus, even though with the correct epithets. This phenomenon confuses researchers who lack taxonomic knowledge, and results in a misapplication of species names to medicinal properties, which then has a negative effect on obtaining permissions from regulatory authorities for commercial development (Zhou 2020).

As stated by Zhou (2020), the use of correct scientific names for fungal species is crucial to studies of traditional Chinese medicine and their commercial exploitation. To facilitate the rational medicinal utilization of Sanghuangporus, all ITS sequences related to "Sanghuang" in GenBank should be re-examined to assist species identification. The aim of the current study is therefore to assess the utility of the ITS region for species discrimination in Sanghuangporus, and reset the species circumscriptions on the basis of the ITS barcoding region, in order to facilitate the correction of previously mislabeled ITS sequences in GenBank, and to provide candidate diagnostic ITS sequences for use in rapid species identification of Sanghuangporus using Hyperbranched Rolling Circle Amplification (HRCA).

\section{MATERIALS AND METHODS}

Morphological examination

The newly sequenced specimens and strains are deposited in HMAS, IFP and BJFC. The specimens were observed with an Olympus BX43 light microscope (Tokyo, Japan) at magnifications up to $1000 \times$. Microscopic procedure followed Zhou et al. (2016). Specimen sections were prepared in Cotton blue (CB), Melzer's reagent (IKI), and $5 \%$ potassium hydroxide $(\mathrm{KOH})$. All measurements were made from material mounted in heated $\mathrm{CB}$. When presenting the variation of basidiospore sizes, $5 \%$ of the measurements were excluded from each end of the range and are given in parentheses. Drawings were made with the aid of a drawing tube. In the text, $\mathrm{L}=$ mean basidiospore length (arithmetic average of all measured basidiospores), $\mathrm{W}=$ mean basidiospore width (arithmetic average 
of all measured basidiospores), $\mathrm{Q}=$ variation in the $\mathrm{L} / \mathrm{W}$ ratios between the studied specimens, and $(\mathrm{a} / \mathrm{b})=$ number of basidiospores (a) measured from given number (b) of specimens.

\section{Molecular sequencing}

A small piece of the basidiome or culture was taken for DNA extraction, which was performed using a CTAB rapid plant genome extraction kit-DN14 (Aidlab Biotechnologies, Beijing). The crude DNA was used as templates for the PCR amplifications of the ITS region. The primer pairs ITS1F/ITS4 and ITS5/ITS4 (White et al. 1990; Gardes and Bruns 1993) were selected for amplification and subsequent sequencing at the Beijing Genomics Institute. The PCR procedure was as follows: initial denaturation at $95^{\circ} \mathrm{C}$ for $3 \mathrm{~min}$, followed by 34 cycles at $94{ }^{\circ} \mathrm{C}$ for $40 \mathrm{~s}, 57.2^{\circ} \mathrm{C}$ for $45 \mathrm{~s}$ and $72{ }^{\circ} \mathrm{C}$ for $1 \mathrm{~min}$, and a final extension at $72^{\circ} \mathrm{C}$ for $10 \mathrm{~min}$. All newly generated sequences are deposited in GenBank (Table 1).

\section{Downloading sequences from GenBank}

The genus name Sanghuangporus and the epithets of 14 Sanghuangporus species were used first as queries to search GenBank. Meanwhile, the reliable sequences of 14 Sanghuangporus species (Zhou et al. 2020) were used as queries to perform BLAST searches in GenBank. The cut-off value of similarity for the resulting sequences was set as $95 \%$. All the ITS sequences matching these queries that had been deposited until 30 April 2020 were retrieved from GenBank (Table 1). In addition, recently published papers related to the taxonomy of Sanghuangporus were checked for supplementary information on collections generating these sequences (Wu et al. 2012a, 2019b; Zhou and Qin 2012; Tian et al. 2013; GhobadNejhad 2015; Tomšovský 2015; Han et al. 2016; Zhou et al. 2016; Zhu et al. 2019; Huo et al. 2020; Shao et al. 2020).

\section{Phylogenetic analyses}

Two datasets of ITS sequences were assembled, one consisting of all sequences recovered from searches of GenBank and newly generated sequences, and the other consisting of the subset of sequences originating from material identified by taxonomists. The datasets were separately aligned using MAFFT 7.110 (Katoh and Standley 2013) under the G-INS-i option (Katoh et al. 2005). All resulting alignments are deposited in TreeBASE (http://www.treebase.org; accession number S26272). jModelTest (Guindon and Gascuel 2003; Posada 2008) was used to estimate the best-fit evolutionary model for each alignment with calculations made under the corrected Akaike information criterion. Following the estimated models, Maximum Likelihood (ML) and Bayesian Inference (BI) algorithms were used to construct midpoint-rooted trees for the alignments. The ML algorithm was performed using raxmlGUI 2.0 (Stamatakis 2014; Edler et al. 2021), and the bootstrap (BS) replicates were calculated under the auto FC option (Pattengale et al. 2010). The BI algorithm was performed using MrBayes 3.2 (Ronquist et al. 2012), which employed two independent runs each with four chains and starting from random trees. Trees were sampled every 1000th generation, of which the first $25 \%$ were removed as burn-in and the other $75 \%$ were retained for constructing a $50 \%$ majority consensus tree and calculating Bayesian posterior probabilities (BPPs). Tracer 1.5 (http:// tree.bio.ed.ac.uk/software/tracer/) was used to judge the convergence of the chains.

\section{Evaluation of molecular species delimitation}

Molecular species delimitation was estimated using multi-rate Poisson Tree Processes (mPTP) method (Kapli et al. 2017). The Newick tree file generated from the ML algorithm was directly uploaded to the web-service version (https://mptp.h-its.org/\#/tree) with no outgroup taxon.

\section{Evaluation of genetic distances of ITS sequences}

The genetic distances of an alignment of ITS sequences were estimated using MEGA X (Kumar et al. 2018; Stecher et al. 2020). For genetic distances between and within species of Sanghuangporus, the parameters were set as follows: a BS method of variance estimation with 1000 BS replications, a $p$-distance substitution model including transitions and transversions, uniform rates among sites, and a pairwise deletion treatment of gaps and missing data.

\section{Identification of diagnostic ITS sequences}

Identification of diagnostic ITS sequences was according to the alignment of the ITS sequences generated using MAFFT 7.110 (Katoh and Standley 2013) under the GINS-i option (Katoh et al. 2005); if a fragment was more than one nucleotide long and was unique for one species and not variant within this species then this fragment was identified as a potential diagnostic sequence for this species.

\section{RESULTS}

A total of 13 specimens and 18 strains were newly sequenced, and the resulting ITS sequences were submitted to GenBank (Table 1). According to our criteria, 240 ITS sequences were downloaded from GenBank, but two sequences (HQ845057 and KP974834, originally identified as Inonotus vaninii and Sanghuangporus baumii, respectively) showed unexpectedly large differences from other sequences of Sanghuangporus by BLAST search, and thus were considered not to belong to the genus and were excluded from subsequent phylogenetic analyses (Table 1). Eventually, a dataset of all available 
Table 1 Information of analyzed ITS sequences of Sanghuangporus

\begin{tabular}{|c|c|c|c|c|c|c|c|c|}
\hline No. & $\begin{array}{l}\text { Species name } \\
\text { accepted here }\end{array}$ & $\begin{array}{l}\text { Species name } \\
\text { in GenBank }\end{array}$ & Voucher No. & GenBank No. & Host plant & $\begin{array}{l}\text { Geographic } \\
\text { origin }\end{array}$ & $\begin{array}{l}\text { Type of } \\
\text { material }\end{array}$ & $\begin{array}{l}\text { Identifier of } \\
\text { material }\end{array}$ \\
\hline 1. & S. alpinus & 1. alpinus & Cui 9646 & $\mathrm{JQ} 860313^{\mathrm{a}}$ & Angiosperm & Tibet, China & Specimen & Tian XM et al. \\
\hline 2. & & 1. alpinus & Cui 9652 & JQ860309 & Angiosperm & Tibet, China & Specimen & Tian XM et al. \\
\hline 3. & & 1. alpinus & Cui 9658 & JQ860310 & Angiosperm & Tibet, China & Specimen & Tian XM et al. \\
\hline 4. & & 1. alpinus & Cui 9666 & JQ860311 & Angiosperm & Tibet, China & Specimen & Tian XM et al. \\
\hline 5. & & S. alpinus & Cui 12444 & $M F 772782^{a}$ & Lonicera & Sichuan, China & Specimen & Zhu L \& Cui BK \\
\hline 6. & & S. alpinus & Cui 12474 & MF772783 & Lonicera & Sichuan, China & Specimen & Zhu L \& Cui BK \\
\hline 7. & & S. alpinus & Cui 12485 & MF $772781^{a}$ & Lonicera & Sichuan, China & Specimen & Zhu L \& Cui BK \\
\hline 8. & & 1. alpinus & Yu 35 & $J Q 860312^{\mathrm{a}}$ & Lonicera & Tibet, China & Specimen & Tian XM et al. \\
\hline 9. & & S. alpinus & Yuan 6396 (IFP) & MT348577 & Lonicera & Qinghai, China & Specimen & This study \\
\hline 10. & & S. alpinus & Yuan 6405 (IFP) & MT348578 & Lonicera & Qinghai, China & Specimen & This study \\
\hline 11. & & S. alpinus & Yuan 6438 (IFP) & MT343579 & Angiosperm & Qinghai, China & Specimen & This study \\
\hline 12. & S. baumii & T. linteus & ASI 26030 & KT 862142 & & South Korea & Strain & Han JG et al. \\
\hline 13. & & T. linteus & ASI 26086 & KT862157 & & $\begin{array}{l}\text { Samchoek, } \\
\text { South Korea }\end{array}$ & Strain & Han JG et al. \\
\hline 14. & & T. linteus & ASI 26087 & KT862158 & & $\begin{array}{l}\text { Mokpo, } \\
\text { South Korea }\end{array}$ & Strain & Han JG et al. \\
\hline 15. & & S. baumii & ASI 26108 & KT862162 & & Inje, South Korea & Strain & Han JG et al. \\
\hline 16. & & 1. baumii & Cui 3573 & $J Q 860307^{a}$ & Syringa & Jilin, China & Specimen & Tian XM et al. \\
\hline 17. & & S. baumii & Cui 11769 & $M F 772784^{a}$ & Angiosperm & $\begin{array}{l}\text { Heilongjiang, } \\
\text { China }\end{array}$ & Specimen & Zhu L \& Cui BK \\
\hline 18. & & S. baumii & Cui 11903 & $\mathrm{KY} 328305^{\mathrm{a}}$ & Alnus & $\begin{array}{l}\text { Heilongjiang, } \\
\text { China }\end{array}$ & Specimen & Zhu L \& Cui BK \\
\hline 19. & & P. baumii & Dai 2340 & AF534069 & & & Strain & Lim YW et al. \\
\hline 20. & & I. baumii & Dai 3683 & $J N 642567^{a}$ & Syringa & $\begin{array}{l}\text { Heilongjiang, } \\
\text { China }\end{array}$ & Strain & Wu SH et al. \\
\hline 21. & & 1. baumii & Dai 3684 & $J N 642568^{a}$ & Syringa & $\begin{array}{l}\text { Heilongjiang, } \\
\text { China }\end{array}$ & Strain & Wu SH et al. \\
\hline 22. & & 1. baumii & Dai 3694 & $J N 642569^{a}$ & Syringa & $\begin{array}{l}\text { Heilongjiang, } \\
\text { China }\end{array}$ & Strain & Wu SH et al. \\
\hline 23. & & S. baumii & Dai 16900 & MF772785 & Syringa & $\begin{array}{l}\text { Heilongjiang, } \\
\text { China }\end{array}$ & Specimen & Zhu L \& Cui BK \\
\hline 24. & & 1. baumii & FS 656165 & HM584807 & & & Strain & Yu TW \\
\hline 25. & & 1. baumii & FS 656164 & GU903007 & & & Strain & Yu TW \\
\hline 26. & & 1. baumii & HLJU & KC312696 & & & Strain & Liu Y et al. \\
\hline 27. & & S. baumii & KUC 10644 & $\mathrm{MH} 168100$ & & & Strain & Heo YM et al. \\
\hline 28. & & 1. baumii & KUC 20130809-20 & KJ668511 & & South Korea & Specimen & Jang Y \& Kim JJ \\
\hline 29. & & 1. baumii & MDJCBS 84 & DQ103887 & & & Strain & Jiang J et al. \\
\hline 30. & & 1. baumii & SFC 050511-32 & AY972811 & & & Strain & Jung HS \& Lee JS \\
\hline 31. & & 1. baumii & SFC 050527-67 & AY972812 & & & Strain & Jung HS \& Lee JS \\
\hline 32. & & P. baumii & SFC 960405-4 & AF534068 & & & Strain & Lim YW et al. \\
\hline 33. & & S. baumii & SFCC 50029 & AY558608 & & & Strain & Jeong WJ et al. \\
\hline 34. & & 1. baumii & $\mathrm{SH} 3$ & FJ190412 & & & Strain & Zou L et al. \\
\hline 35. & & S. baumii & Yuan 4909 & $\mathrm{KY} 328310^{\mathrm{a}}$ & Angiosperm & $\begin{array}{l}\text { Heilongjiang, } \\
\text { China }\end{array}$ & Specimen & Zhu L \& Cui BK \\
\hline 36. & & S. baumii & Yuan 4929 & KY328306 & Alnus & $\begin{array}{l}\text { Heilongjiang, } \\
\text { China }\end{array}$ & Specimen & Zhu L \& Cui BK \\
\hline 37. & S. ligneus & S. ligneus & MG 12 & $\mathrm{KR} 073081^{\mathrm{a}}$ & Lonicera caucasica & Iran & Strain & Ghobad-Nejhad M \\
\hline 38. & & S. ligneus & MG 13 & KR073082 ${ }^{\mathrm{a}}$ & Lonicera caucasica & Iran & Strain & Ghobad-Nejhad M \\
\hline 39. & S. Ionicericola & 1. baumii & BM-3753 & HQ845063 & & China & Strain & Hu W \& Deng X \\
\hline
\end{tabular}


Table 1 Information of analyzed ITS sequences of Sanghuangporus (Continued)

\begin{tabular}{|c|c|c|c|c|c|c|c|c|}
\hline No. & $\begin{array}{l}\text { Species name } \\
\text { accepted here }\end{array}$ & $\begin{array}{l}\text { Species name } \\
\text { in GenBank }\end{array}$ & Voucher No. & GenBank No. & Host plant & $\begin{array}{l}\text { Geographic } \\
\text { origin }\end{array}$ & $\begin{array}{l}\text { Type of } \\
\text { material }\end{array}$ & $\begin{array}{l}\text { Identifier of } \\
\text { material }\end{array}$ \\
\hline 40. & & 1. baumii & BM-8335 & HQ845064 & & China & Strain & Hu W \& Deng X \\
\hline 41. & & S. Ionicericola & Cui 10994 & $M F 772786^{a}$ & & China & Specimen & Zhu L \& Cui BK \\
\hline 42. & & I. Ionicericola & Dai 8322 & JN642571 ${ }^{\mathrm{a}}$ & Lonicera & Heilongjiang, China & Specimen & Wu SH et al. \\
\hline 43. & & 1. Ionicericola & Dai 8335 & JN642573 & Lonicera & Heilongjiang, China & Specimen & Wu SH et al. \\
\hline 44. & & I. Ionicericola & Dai 8340 & JN642574 & Lonicera & Heilongjiang, China & Specimen & Wu SH et al. \\
\hline 45. & & 1. Ionicericola & Dai 8376 & $J Q 860308^{a}$ & Lonicera & Heilongjiang, China & Specimen & Tian XM et al. \\
\hline 46. & & S. lonicericola & Dai 17304 (BJFC) & MT348582 $^{\mathrm{a}}$ & Lonicera & Liaoning, China & Strain & This study \\
\hline 47. & & P. sp. & HN100K9 & KF589300 & & South Korea & Strain & $\begin{array}{l}\text { Kang HW \& Kim } \\
\text { JK }\end{array}$ \\
\hline 48. & & P. ribis & SFCC 50032 & AY558643 & & & Strain & Jeong WJ et al. \\
\hline 49. & & I. Ionicericola & TAA 105317 & $\mathrm{JN} 642572^{\mathrm{a}}$ & $\begin{array}{l}\text { Lonicera } \\
\text { ruprechtiana }\end{array}$ & Russian Far East & Specimen & Wu SH et al. \\
\hline 50. & S. lonicerinus & S. Ionicerinus & Dai 17093 & $M F 772788^{a}$ & Lonicera & Uzbekistan & Specimen & Zhu L \& Cui BK \\
\hline 51. & & S. Ionicerinus & Dai 17095 & MF772787 & Lonicera & Uzbekistan & Specimen & Zhu L \& Cui BK \\
\hline 52. & & S. Ionicerinus & MG 280 & $\mathrm{KU} 213573^{\mathrm{a}}$ & & & Specimen & $\begin{array}{l}\text { Langer EJ \& } \\
\text { Ghobad-Nejhad M }\end{array}$ \\
\hline 53. & & S. lonicerinus & MG 281 & KU213574 & & & Specimen & $\begin{array}{l}\text { Langer EJ \& } \\
\text { Ghobad-Nejhad M }\end{array}$ \\
\hline 54. & & I. sp. & TAA 55428 & JN642575 & Lonicera & Turkmenistan & Strain & Wu SH et al. \\
\hline 55. & & S. Ionicerinus & TAA 55696 & MT348583 $^{\mathrm{a}}$ & Lonicera & Turkmenistan & Specimen & This study \\
\hline 56. & & P. linteus & TAA-104264 & AF534074 & & & Strain & Lim YW et al. \\
\hline 57. & S. microcystideus & $\begin{array}{l}\text { S. } \\
\text { microcystideus }\end{array}$ & O 915609 & $\mathrm{KP} 030787^{\mathrm{a}}$ & Olea africana & Tanzania & Specimen & Zhou LW et al. \\
\hline 58. & S. pilatii & P. pilatii & BRNM 771989 & KT428764 & Populus alba & Czech Republic & Specimen & Tomšovský M \\
\hline 59. & S. quercicola & $\begin{array}{l}\text { P. } \\
\text { rhabarbarinus }\end{array}$ & CBS 282.77 & AY558642 & & & Strain & Jeong WJ et al. \\
\hline 60. & & S. quercicola & Dai 13947 & KY328309a & & Chongqing, China & Specimen & Zhu L \& Cui BK \\
\hline 61. & & S. quercicola & Li 445 & $\mathrm{KY} 328311^{\mathrm{a}}$ & Angiosperm & Henan, China & Specimen & Zhu L \& Cui BK \\
\hline 62. & & S. quercicola & Li 1149 & $\mathrm{KY} 328312^{\mathrm{a}}$ & Quercus & Henan, China & Specimen & Zhu L \& Cui BK \\
\hline 63. & & S. quercicola & LWZ 20170821-13 (IFP) & MT348584 & Angiosperm & Hubei, China & Specimen & This study \\
\hline 64. & & S. quercicola & LWZ 20170821-14 (IFP) & MT348585 & Angiosperm & Hubei, China & Specimen & This study \\
\hline 65. & & S. quercicola & LWZ 20170821-18 (IFP) & MT348586 ${ }^{\mathrm{a}}$ & Angiosperm & Hubei, China & Specimen & This study \\
\hline 66. & & S. quercicola & Wei 7575 (IFP) & MT348587 & Quercus & Henan, China & Strain & This study \\
\hline 67. & & S. sp. & Wu 1805-2 & MK400422 & Toxicodendron & Hubei, China & Specimen & Wu SH et al. \\
\hline 68. & & S. sp. & Wu 1805-3 & MK400423 a & Toxicodendron & Hubei, China & Specimen & Wu SH et al. \\
\hline 69. & & S. sp. & Wu $1805-5$ & MK400424 & Toxicodendron & Hubei, China & Specimen & Wu SH et al. \\
\hline 70. & & S. sp. & Wu 1807-2 & MK729538 & Toxicodendron & Hubei, China & Specimen & Wu SH et al. \\
\hline 71. & & S. sp. & Wu 1807-3 & MK729540 a & Toxicodendron & Hubei, China & Specimen & Wu SH et al. \\
\hline 72. & & S. sp. & Wu 1807-4 & MK729539 & Toxicodendron & Hubei, China & Specimen & Wu SH et al. \\
\hline 73. & S. sanghuang & 1. baumii & & KM385537 & & Viet Nam & Strain & $\begin{array}{l}\text { Hanh W \& } \\
\text { Nguyet NT }\end{array}$ \\
\hline 74. & & S. sanghuang & AH1 (HMAS) & MT421899 ${ }^{\mathrm{a}}$ & Cultivated & Anhui, China & Strain & This study \\
\hline 75. & & S. sanghuang & AH2 (HMAS) & MT421900 & Cultivated & Anhui, China & Strain & This study \\
\hline 76. & & S. sanghuang & AH3 (HMAS) & MT421901 ${ }^{a}$ & Cultivated & Anhui, China & Strain & This study \\
\hline 77. & & S. sanghuang & AH4 (HMAS) & MT421902 & Cultivated & Anhui, China & Strain & This study \\
\hline 78. & & S. sanghuang & AH5 (HMAS) & MT421903 ${ }^{a}$ & Cultivated & Anhui, China & Strain & This study \\
\hline 79. & & P. igniarius & ASI 26010 & KT862134 & & $\begin{array}{l}\text { Jeongseon, } \\
\text { South Korea }\end{array}$ & Strain & Han JG et al. \\
\hline
\end{tabular}


Table 1 Information of analyzed ITS sequences of Sanghuangporus (Continued)

\begin{tabular}{|c|c|c|c|c|c|c|c|c|}
\hline No. & $\begin{array}{l}\text { Species name } \\
\text { accepted here }\end{array}$ & $\begin{array}{l}\text { Species name } \\
\text { in GenBank }\end{array}$ & Voucher No. & GenBank No. & Host plant & $\begin{array}{l}\text { Geographic } \\
\text { origin }\end{array}$ & $\begin{array}{l}\text { Type of } \\
\text { material }\end{array}$ & $\begin{array}{l}\text { Identifier of } \\
\text { material }\end{array}$ \\
\hline 80. & & T. linteus & ASI 26011 & KT 862135 & & India & Strain & Han JG et al. \\
\hline 81. & & T. linteus & ASI 26016 & KT 862136 & & South Korea & Strain & Han JG et al. \\
\hline 82. & & T. linteus & ASI 26021 & KT862138 & & $\begin{array}{l}\text { Hongcheon, } \\
\text { South Korea }\end{array}$ & Strain & Han JG et al. \\
\hline 83. & & T. linteus & ASI 26022 & KT862139 & & $\begin{array}{l}\text { Hongcheon, } \\
\text { South Korea }\end{array}$ & Strain & Han JG et al. \\
\hline 84. & & T. linteus & ASI 26025 & KT 862140 & & $\begin{array}{l}\text { Wonju, } \\
\text { South Korea }\end{array}$ & Strain & Han JG et al. \\
\hline 85. & & T.linteus & ASI 26026 & KT862141 & & $\begin{array}{l}\text { Wonju, } \\
\text { South Korea }\end{array}$ & Strain & Han JG et al. \\
\hline 86. & & T. linteus & ASI 26039 & KT 862143 & & $\begin{array}{l}\text { Pyeongchang, } \\
\text { South Korea }\end{array}$ & Strain & Han JG et al. \\
\hline 87. & & T. linteus & ASI 26046 & KT862144 & & $\begin{array}{l}\text { Hongcheon, } \\
\text { South Korea }\end{array}$ & Strain & Han JG et al. \\
\hline 88. & & T. linteus & ASI 26049 & KT 862145 & & $\begin{array}{l}\text { Hongcheon, } \\
\text { South Korea }\end{array}$ & Strain & Han JG et al. \\
\hline 89. & & T. linteus & ASI 26054 & KT862147 & & $\begin{array}{l}\text { Hongcheon, } \\
\text { South Korea }\end{array}$ & Strain & Han JG et al. \\
\hline 90. & & T. linteus & ASI 26062 & KT 862148 & & $\begin{array}{l}\text { Hwacheon, } \\
\text { South Korea }\end{array}$ & Strain & Han JG et al. \\
\hline 91. & & T. linteus & ASI 26063 & KT862149 & & $\begin{array}{l}\text { Jeongseon, } \\
\text { South Korea }\end{array}$ & Strain & Han JG et al. \\
\hline 92. & & T. linteus & ASI 26066 & KT862150 & & Inje, South Korea & Strain & Han JG et al. \\
\hline 93. & & T. linteus & ASI 26067 & KT862151 & & Inje, South Korea & Strain & Han JG et al. \\
\hline 94. & & T. linteus & ASI 26070 & KT862152 & & & Strain & Han JG et al. \\
\hline 95. & & T. linteus & ASI 26071 & KT862153 & & & Strain & Han JG et al. \\
\hline 96. & & T. linteus & ASI 26073 & KT862154 & & South Korea & Strain & Han JG et al. \\
\hline 97. & & T. linteus & ASI 26074 & KT 862155 & & $\begin{array}{l}\text { Seongnam, } \\
\text { South Korea }\end{array}$ & Strain & Han JG et al. \\
\hline 98. & & T. linteus & ASI 26082 & KT 862156 & & $\begin{array}{l}\text { Mokpo, } \\
\text { South Korea }\end{array}$ & Strain & Han JG et al. \\
\hline 99. & & T. linteus & ASI 26088 & KT862159 & & $\begin{array}{l}\text { Sancheong, } \\
\text { South Korea }\end{array}$ & Strain & Han JG et al. \\
\hline 100. & & T. linteus & ASI 26114 & KT 862164 & & South Korea & Strain & Han JG et al. \\
\hline 101. & & T. linteus & ASI 26115 & KT 862165 & & South Korea & Strain & Han JG et al. \\
\hline 102. & & P. linteus & ATCC 26710 & AF153010 & & South Korea & Strain & Kim GY et al. \\
\hline 103. & & S. sanghuang & Batch 1-12192170-1 & KT693244 & Purchased & USA & Strain & Raja HA et al. \\
\hline 104. & & S. sanghuang & Batch 2-10221252-2 & KT693275 & Purchased & USA & Strain & Raja HA et al. \\
\hline 105. & & S. sanghuang & Batch 2-12192170-1 & KT693246 & Purchased & USA & Strain & Raja HA et al. \\
\hline 106. & & S. sanghuang & BJ (HMAS) & MT421904 & Cultivated & Beijing, China & Strain & This study \\
\hline 107. & & 1. sp. & $B Z-A$ & JN642589a & Morus & Hunan, China & Strain & Wu SH et al. \\
\hline 108. & & I. sp. & $B Z-C$ & JN642587 & Morus & Hunan, China & Strain & Wu SH et al. \\
\hline 109. & & 1. sp. & CA & $J N 642579^{a}$ & Morus & Jiangxi, China & Strain & Wu SH et al. \\
\hline 110. & & 1. sp. & $C B$ & JN642580 & Morus & Jiangxi, China & Strain & Wu SH et al. \\
\hline 111. & & 1. sp. & $\mathrm{CC}$ & $J N 642581^{a}$ & Morus & Jiangxi, China & Strain & Wu SH et al. \\
\hline 112. & & S. sanghuang & Cui 14419 & MF772789 & Morus & Shaanxi, China & Specimen & Zhu L \& Cui BK \\
\hline 113. & & S. sanghuang & Cui 14420 & MF772790 & Morus & Shaanxi, China & Specimen & Zhu L \& Cui BK \\
\hline 114. & & 1. sanghuang & Dai 12723 & JQ860316 & Morus & Sichuan, China & Specimen & Tian XM et al. \\
\hline 115. & & S. sanghuang & DB1 (HMAS) & MT421905 & Cultivated & Northeast China & Strain & This study \\
\hline 116. & & P. linteus & DGUM25003 & AF082102 & & & Strain & Chung JW et al. \\
\hline
\end{tabular}


Table 1 Information of analyzed ITS sequences of Sanghuangporus (Continued)

\begin{tabular}{|c|c|c|c|c|c|c|c|c|}
\hline No. & $\begin{array}{l}\text { Species name } \\
\text { accepted here }\end{array}$ & $\begin{array}{l}\text { Species name } \\
\text { in GenBank }\end{array}$ & Voucher No. & GenBank No. & Host plant & $\begin{array}{l}\text { Geographic } \\
\text { origin }\end{array}$ & $\begin{array}{l}\text { Type of } \\
\text { material }\end{array}$ & $\begin{array}{l}\text { Identifier of } \\
\text { material }\end{array}$ \\
\hline 117. & & P. linteus & DGUM25004 & AF080458 & & & Strain & Chung JW et al. \\
\hline 118. & & 1. linteus & FS 656160 & GU903004 & & & Strain & Yu TW \\
\hline 119. & & 1. linteus & FS 656161 & HM584806 & & & Strain & Yu TW \\
\hline 120. & & T. linteus & FS 656179 & KU867779 & & & Strain & Yu TW \\
\hline 121. & & T. linteus & FS 656180 & KU867780 & & & Strain & Yu TW \\
\hline 122. & & S. sanghuang & HB (HMAS) & MT421907 & Cultivated & Hubei, China & Strain & This study \\
\hline 123. & & P. linteus & IFO 6980 & AF200226 & & & Strain & Kim GY \& Lee JD \\
\hline 124. & & I. linteus & IFO 6989 & AY640937 & & & Strain & Lee JS \& Jung HS \\
\hline 125. & & P. linteus & IMSNU 31014 & AF082101 & & & Strain & Chung JW et al. \\
\hline 126. & & S. sanghuang & $J L-01$ & MG062789 & & & Strain & $\mathrm{XuX}$ \\
\hline 127. & & S. sanghuang & JS1 (HMAS) & MT421908 & Cultivated & Jiangsu, China & Strain & This study \\
\hline 128. & & 1. linteus & KAB-PL-01 & DQ462333 & & Taiwan, China & Strain & Chiou SJ \& Yen J! \\
\hline 129. & & P. linteus & KCTC 6190 & AF077678 & & & Strain & Chung JW et al. \\
\hline 130. & & P. igniarius & KCTC 16890 & AY189708 & & & Strain & Nam BH et al. \\
\hline 131. & & I. linteus & KFDA 016 & AY436626 & & & Strain & Yun JC et al. \\
\hline 132. & & I. linteus & KFDA P38 & AY513234 & & & Strain & Jin CY et al. \\
\hline 133. & & I. linteus & KSSW01 & EF506943 & & & Strain & Park SY et al. \\
\hline 134. & & 1. linteus & LT-0802 & HQ845059 & & South Korea & Strain & Hu W \& Deng X \\
\hline 135. & & 1. linteus & LT-CBS83 & HQ845060 & & South Korea & Strain & Hu W \& Deng X \\
\hline 136. & & S. sanghuang & $\begin{array}{l}\text { LWZ 20180927-3 } \\
\text { (HMAS) }\end{array}$ & MT348588 & Morus & Yunnan, China & Specimen & This study \\
\hline 137. & & P. linteus & MPNU 7016 & AF153009 & & & Strain & Kim GY et al. \\
\hline 138. & & 1. linteus & MUCL 47139 & GU461973 & & Cuba & Strain & Amalfi M et al. \\
\hline 139. & & 1. linteus & NAAS00002 & JN043317 & & & Strain & Seok SJ et al. \\
\hline 140. & & P. linteus & Namsan No1 & AF080457 & & & Strain & Chung JW et al. \\
\hline 141. & & 1. linteus & PL 0801 & FJ940906 & & & Strain & Xie LY et al. \\
\hline 142. & & I. linteus & PL 5 & EF095712 & & & Strain & Park BW et al. \\
\hline 143. & & I. sp. & PL 10 & JN642588 & & China & Strain & Wu SH et al. \\
\hline 144. & & S. sanghuang & S3 & MN153568 & & & Strain & Song JL et al. \\
\hline 145 & & P. sp. & SA 01 & EF694971 & & & Strain & Zeng NK et al. \\
\hline 146. & & P. baumii & SFC 20001106-1 & AF534064 & & & Strain & Lim YW et al. \\
\hline 147. & & P. baumii & SFC 20010212-1 & AF534062 & & & Strain & Lim YW et al. \\
\hline 148. & & S. sanghuang & SS & MG209821 & & & Strain & Cai C \& Zhao G \\
\hline 149. & & I. sp. & T004 & JN642586 & Morus & Taiwan, China & Strain & Wu SH et al. \\
\hline 150. & & I. sp. & $\mathrm{TH}$ & JN642582 & Morus & Taiwan, China & Strain & Wu SH et al. \\
\hline 151. & & I. sp. & TJ & $J N 642585^{a}$ & Morus & Taiwan, China & Strain & Wu SH et al. \\
\hline 152. & & I. sp. & TM & JN642583 & Morus & Taiwan, China & Strain & Wu SH et al. \\
\hline 153. & & I. sp. & TN & $J N 642584^{a}$ & Morus & Taiwan, China & Strain & Wu SH et al. \\
\hline 154. & & I. sp. & WD 1222 & $J N 642576^{a}$ & Morus & Japan & Strain & Wu SH et al. \\
\hline 155. & & I. sp. & WD 2261 & $J N 642577^{a}$ & Morus & Japan & Strain & Wu SH et al. \\
\hline 156. & & I. sp. & WD 2300 & JN642578 & Morus & Japan & Strain & Wu SH et al. \\
\hline 157. & & I. sp. & Wu 0903-1 & JN794061 & Morus & Jilin, China & Strain & Wu SH et al. \\
\hline 158. & & I. sp. & ZhangjiaJie & MN242716 & Cultivated & & Strain & Wang $Y$ \\
\hline 159. & & S. sanghuang & ZJ1 (HMAS) & MT421910 & Cultivated & Zhejiang, China & Strain & This study \\
\hline 160. & & S. sanghuang & ZJ2 (HMAS) & MT421911 & Cultivated & Zhejiang, China & Strain & This study \\
\hline
\end{tabular}


Table 1 Information of analyzed ITS sequences of Sanghuangporus (Continued)

\begin{tabular}{|c|c|c|c|c|c|c|c|c|}
\hline No. & $\begin{array}{l}\text { Species name } \\
\text { accepted here }\end{array}$ & $\begin{array}{l}\text { Species name } \\
\text { in GenBank }\end{array}$ & Voucher No. & GenBank No. & Host plant & $\begin{array}{l}\text { Geographic } \\
\text { origin }\end{array}$ & $\begin{array}{l}\text { Type of } \\
\text { material }\end{array}$ & $\begin{array}{l}\text { Identifier of } \\
\text { material }\end{array}$ \\
\hline 161. & & S. sanghuang & ZJ4 (HMAS) & MT421913 $^{a}$ & Cultivated & Zhejiang, China & Strain & This study \\
\hline 162. & & S. sanghuang & ZJ5 (HMAS) & MT421914 & Cultivated & Zhejiang, China & Strain & This study \\
\hline 163. & S. subbaumii & 1. baumii & BZ-2029 & JN642565 & Pruchased & China & Strain & Wu SH et al. \\
\hline 164. & & 1. baumii & BZ-2030 & JN642566 & Pruchased & China & Strain & Wu SH et al. \\
\hline 165. & & S. subbaumii & Dai 13360 (BJFC) & MT343580 & Prunus & Shanxi, China & Specimen & This study \\
\hline 166. & & S. subbaumii & $\begin{array}{l}\text { LWZ 20190722-18 } \\
\text { (HMAS) }\end{array}$ & MT348581 & Angiosperm & Beijing, China & Specimen & This study \\
\hline 167. & & P. linteus & SFC 970527-1 & AF534073 & & & Strain & Lim YW et al. \\
\hline 168. & & 1. baumii & Wu 0910-54 & $\mathrm{JN} 642570^{\mathrm{a}}$ & Syringa & Beijing, China & Strain & Wu SH et al. \\
\hline 169. & & 1. baumii & Yuan 2444 & JX069836a & Angiosperm & Shanxi, China & Specimen & Tian XM et al. \\
\hline 170. & S. vaninii & I. vaninii & & HQ845058 & & China & Strain & Hu W \& Deng X \\
\hline 171. & & I. sp. & Beiling & MN242720 & Cultivated & China & Strain & Wang $Y$ \\
\hline 172. & & I. vaninii & BZ-2031 & $J N 642593^{a}$ & Populus & China & Strain & Wu SH et al. \\
\hline 173. & & I. vaninii & CJC 01 & $J N 642592^{a}$ & Cultivated & Taiwan, China & Strain & Wu SH et al. \\
\hline 174. & & S. vaninii & Cui 9939 & MF772792 & & Jilin, China & Specimen & Zhu L \& Cui BK \\
\hline 175. & & S. vaninii & Cui 14082 & MF772793 & Populus & Jilin, China & Specimen & Zhu L \& Cui BK \\
\hline 176. & & I. vaninii & Dai 3624 & JN642590 & Populus & China & Strain & Wu SH et al. \\
\hline 177. & & I. vaninii & Dai 7011 & JN642591 & Populus davidiana & Jilin, China & Strain & Wu SH et al. \\
\hline 178. & & S. vaninii & Dai 8236 & MF772791 & Populus & Jilin, China & Specimen & Zhu L \& Cui BK \\
\hline 179. & & S. vaninii & DB2 (HMAS) & MT421906 & Cultivated & Northeast China & Strain & This study \\
\hline 180. & & 1. baumii & FS 656170 & GU903008 & & & Strain & Yu TW \\
\hline 181. & & F. gilva & FS 656175 & HM584811 & & & Strain & Yu TW \\
\hline 182. & & S. vaninii & $\mathrm{HZ}-01$ & MG062791 & & & Strain & XuX \\
\hline 183. & & I. sp. & JinZhai & MN242717 & Cultivated & China & Strain & Wang $Y$ \\
\hline 184. & & S. vaninii & JS2 (HMAS) & MT421909 & Cultivated & Jiangsu, China & Strain & This study \\
\hline 185. & & I. sp. & KangNeng & MN242721 & Cultivated & China & Strain & Wang $Y$ \\
\hline 186. & & 1. baumii & KFDA 015 & AY436623 & & & Strain & Yun JC et al. \\
\hline 187. & & 1. baumii & KFDA 022 & AY436624 & & & Strain & Yun JC et al. \\
\hline 188. & & I. linteus & KFDA 024 & AY436627 & & & Strain & Yun JC et al. \\
\hline 189. & & 1. baumii & KFDA 029 & AY436625 & & & Strain & Yun JC et al. \\
\hline 190. & & 1. baumii & KFDA P36 & AY509198 & & & Strain & Jin CY et al. \\
\hline 191. & & 1. baumii & KFDA P40 & AY509199 & & & Strain & Jin CY et al. \\
\hline 192. & & 1. baumii & KFDA P45 & AY509201 & & & Strain & Jin $C Y$ et al. \\
\hline 193. & & I. sp. & Korea & MN242719 & Cultivated & China & Strain & Wang $Y$ \\
\hline 194. & & S. baumii & LC 6686 & MK818502 & & & Strain & Li ZN \\
\hline 195. & & I. linteus & LT-HG & HQ845061 & & & Strain & Hu W \& Deng X \\
\hline 196. & & F. gilva & MDJCBS87 & DQ103884 & & & Strain & Jiang J et al. \\
\hline 197. & & P. baumi & MPNU 7004 & AF200229 & & & Strain & Kim GY \& Lee JD \\
\hline 198. & & P. baumi & MPNU 7005 & AF200230 & & & Strain & Kim GY \& Lee JD \\
\hline 199. & & P. baumi & MPNU 7006 & AF200231 & & & Strain & Kim GY \& Lee JD \\
\hline 200. & & P. sp. & MPNU 7007 & AF200235 & & & Strain & Kim GY \& Lee JD \\
\hline 201. & & P. sp. & MPNU 7010 & AF153007 & & South Korea & Strain & Kim GY et al. \\
\hline 202. & & P. sp. & MPNU 7012 & AF153008 & & South Korea & Strain & Kim GY et al. \\
\hline 203. & & P. sp. & MPNU 7013 & AF153011 & & South Korea & Strain & Kim GY et al. \\
\hline 204. & & I. baumii & PB 0802 & FJ940907 & & & Strain & Xie LY et al. \\
\hline
\end{tabular}


Table 1 Information of analyzed ITS sequences of Sanghuangporus (Continued)

\begin{tabular}{|c|c|c|c|c|c|c|c|c|}
\hline No. & $\begin{array}{l}\text { Species name } \\
\text { accepted here }\end{array}$ & $\begin{array}{l}\text { Species name } \\
\text { in GenBank }\end{array}$ & Voucher No. & GenBank No. & Host plant & $\begin{array}{l}\text { Geographic } \\
\text { origin }\end{array}$ & $\begin{array}{l}\text { Type of } \\
\text { material }\end{array}$ & $\begin{array}{l}\text { Identifier of } \\
\text { material }\end{array}$ \\
\hline 205. & & 1. baumii & PB 0803 & FJ940908 & & & Strain & Xie LY et al. \\
\hline 206. & & 1. baumii & PB 0806 & FJ940911 & & & Strain & Xie LY et al. \\
\hline 207. & & 1. baumii & PB 0808 & FJ940913 & & & Strain & Xie LY et al. \\
\hline 208. & & 1. baumii & PB 0809 & FJ940914 & & & Strain & Xie LY et al. \\
\hline 209. & & I. sp. & QianDaoHu & MN242718 & Cultivated & China & Strain & Wang $Y$ \\
\hline 210. & & S. vaninii & S1 & MN153566 & & & Strain & Song $J \mathrm{~L}$ et al. \\
\hline 211. & & S. baumii & S2 & MN153567 & & & Strain & Song $J \mathrm{~L}$ et al. \\
\hline 212. & & F. gilva & $\mathrm{S} 12$ & MT275660 & Morus & Zhejiang, China & Strain & Li Y \& Huo J \\
\hline 213. & & P. sp. & SA 02 & EF694972 & & & Strain & Zeng NK et al. \\
\hline 214. & & P. sp. & SA 03 & EF694973 & & & Strain & Zeng NK et al. \\
\hline 215. & & P. sp. & SA 04 & EF694974 & & & Strain & Zeng NK et al. \\
\hline 216. & & 1. baumii & SA 05 & EF694975 & & & Strain & Zeng NK et al. \\
\hline 217. & & P. sp. & SA 06 & EF694976 & & & Strain & Zeng NK et al. \\
\hline 218. & & P. sp. & SA 07 & EF694977 & & & Strain & Zeng NK et al. \\
\hline 219. & & P. linteus & SFC 970605 & AF534071 & & & Strain & Lim YW et al. \\
\hline 220. & & P. linteus & SFC 20001106-7 & AF534070 & & & Strain & Lim YW et al. \\
\hline 221. & & P. baumii & SFC 20010212-2 & AF534063 & & & Strain & Lim YW et al. \\
\hline 222. & & T. linteus & SFCC 10209 & AY558628 & & & Strain & Jeong WJ et al. \\
\hline 223. & & F. gilva & $\mathrm{SH} 1$ & FJ190410 & & & Strain & Zou L et al. \\
\hline 224. & & 1. baumii & SJ & JN887691 & & & Strain & Shin KS \\
\hline 225. & & I. vaninii & Wei 3382 & JN169788 & & Jilin, China & Specimen & $\begin{array}{l}\text { Zhou LW \& } \\
\text { Qin WM }\end{array}$ \\
\hline 226. & & I. vaninii & WN 0801 & HQ845054 & & China & Strain & Hu W \& Deng X \\
\hline 227. & & I. vaninii & WN-1 & HQ845055 & & China & Strain & Hu W \& Deng X \\
\hline 228. & & I. vaninii & $W N-2$ & HQ845056 & & China & Strain & Hu W \& Deng X \\
\hline 229. & & I. vaninii & WN-4 & HQ845065 & & China & Strain & Hu W \& Deng X \\
\hline 230. & & I. vaninii & WN 8213 & HQ845052 & & China & Strain & Hu W \& Deng X \\
\hline 231. & & I. vaninii & WN 8824 & HQ845051 & & China & Strain & Hu W \& Deng X \\
\hline 232. & & I. vaninii & WN 3624 & HQ845050 & & China & Strain & Hu W \& Deng X \\
\hline 233. & & S. baumii & $X Z-01$ & MG062790 & & & Strain & XuX \\
\hline 234. & & 1. baumii & YC & JN887692 & & & Strain & Shin KS \\
\hline 235. & & S. vaninii & Yuan 2764 & $K Y 328308^{a}$ & Quercus & Shaanxi, China & Specimen & Zhu L \& Cui BK \\
\hline 236. & & S. vaninii & Yuan 5604 & $K Y 328307^{a}$ & Quercus & Jilin, China & Specimen & Zhu L \& Cui BK \\
\hline 237. & & S. vaninii & ZJ3 (HMAS) & MT421912 & Cultivated & Zhejiang, China & Strain & This study \\
\hline 238. & S. weigelae & S. weigelae & 420526MF0201 & MH142013 & & Hubei, China & Specimen & Wang $R$ et al. \\
\hline 239. & & 1. weigelae & Cui 6010 & $J Q 860318^{a}$ & Lonicera & Jiangxi, China & Specimen & Tian XM et al. \\
\hline 240. & & 1. weigelae & Cui 6012 & $J Q 860319^{a}$ & Lonicera & Jiangxi, China & Specimen & Tian XM et al. \\
\hline 241. & & 1. weigelae & Cui 7176 & $J Q 860320^{\mathrm{a}}$ & Syringa & Hebei, China & Specimen & Tian XM et al. \\
\hline 242. & & 1. weigelae & Dai 6352 & $\mathrm{JQ} 860317^{\mathrm{a}}$ & & Zhejiang, China & Specimen & Tian XM et al. \\
\hline 243. & & 1. weigelae & Dai 11694 & $\mathrm{JQ} 860315^{\mathrm{a}}$ & & Hunan, China & Specimen & Tian XM et al. \\
\hline 244. & & S. weigelae & Dai 15770 & MF772795 & Weigela & Chongqing, China & Specimen & Zhu L \& Cui BK \\
\hline 245. & & S. weigelae & Dai 16072 (BJFC) & MT348589 & Weigela & $\begin{array}{l}\text { Inner Mongolia, } \\
\text { China }\end{array}$ & Specimen & This study \\
\hline 246. & & S. weigelae & Dai 16077 & $M F 772794^{a}$ & Weigela & $\begin{array}{l}\text { Inner Mongolia, } \\
\text { China }\end{array}$ & Specimen & Zhu L \& Cui BK \\
\hline 247. & & S. weigelae & LWZ 20150802-3 (IFP) & MT348590 & Weigela & Jiangxi, China & Specimen & This study \\
\hline
\end{tabular}


Table 1 Information of analyzed ITS sequences of Sanghuangporus (Continued)

\begin{tabular}{|c|c|c|c|c|c|c|c|c|}
\hline No. & $\begin{array}{l}\text { Species name } \\
\text { accepted here }\end{array}$ & $\begin{array}{l}\text { Species name } \\
\text { in GenBank }\end{array}$ & Voucher No. & GenBank No. & Host plant & $\begin{array}{l}\text { Geographic } \\
\text { origin }\end{array}$ & $\begin{array}{l}\text { Type of } \\
\text { material }\end{array}$ & $\begin{array}{l}\text { Identifier of } \\
\text { material }\end{array}$ \\
\hline 248. & & S. weigelae & LWZ 20150802-5 (IFP) & MT348591 $^{\mathrm{a}}$ & Weigela & Jiangxi, China & Specimen & This study \\
\hline 249. & & P. baumii & SFC 20000111-10 & AF534067 & & & Strain & Lim YW et al. \\
\hline 250. & & 1. sp. & WD 1186 & JN642597 & Weigela & Japan & Strain & Tian XM et al. \\
\hline 251. & & I. sp. & WD 1187 & $J N 642598^{\mathrm{a}}$ & Weigela & Japan & Strain & Tian XM et al. \\
\hline 252. & & 1. sp. & WD 1667 & $J N 642594^{a}$ & $\begin{array}{l}\text { Weigela } \\
\text { cordeenis }\end{array}$ & Japan & Strain & Wu SH et al. \\
\hline 253. & & I. sp. & WD 1837 & $J \mathrm{~N} 642595^{\mathrm{a}}$ & $\begin{array}{l}\text { Weigela } \\
\text { cordeenis }\end{array}$ & Japan & Strain & Wu SH et al. \\
\hline 254. & & 1. sp. & WD 1838 & $J \mathrm{~N} 642596^{\mathrm{a}}$ & $\begin{array}{l}\text { Weigela } \\
\text { cordeenis }\end{array}$ & Japan & Strain & Wu SH et al. \\
\hline 255. & & 1. weigelae & Wei 2120 & JQ860314 & Coriaria & Hubei, China & Specimen & Tian XM et al. \\
\hline 256. & & 1. weigelae & Wei 2267 & $J \times 069835^{a}$ & Angiosperm & Hubei, China & Specimen & Tian XM et al. \\
\hline 257. & & $\begin{array}{l}\text { I. } \\
\text { tenuicontextus }\end{array}$ & Yuan 5526 & $J N 169786^{a}$ & Angiosperm & Guizhou, China & Specimen & $\begin{array}{l}\text { Zhou LW \& } \\
\text { Qin WM }\end{array}$ \\
\hline 258. & S. weirianus & S. weirianus & CBS 618.89 & AY558654 & Juglans major & Arizona, USA & Strain & Jeong WJ et al. \\
\hline 259. & & P. weirianus & IMSNU 32021 & AF110989a & Juglans major & Arizona, USA & Strain & Chung JW et al. \\
\hline 260. & S. zonatus & 1. zonatus & Cui 6631 & $\mathrm{JQ} 860305^{\mathrm{a}}$ & Angiosperm & Hainan, China & Specimen & Tian XM et al. \\
\hline 261. & & 1. zonatus & Cui 8327 & JX069837 & Angiosperm & Yunnan, China & Specimen & Tian XM et al. \\
\hline 262. & & I. zonatus & Dai 10841 & $J Q 860306^{\mathrm{a}}$ & Angiosperm & Hainan, China & Specimen & Tian XM et al. \\
\hline 263. & S. sp. 1 & I. sp. & AM-08 & JF895464 & & Ethiopia & Specimen & Assefa A et al. \\
\hline 264. & & I. sp. & AM-19 & JF895465 & & Ethiopia & Specimen & Assefa A et al. \\
\hline 265. & & I. linteus & F915611 & JX985739 & & Ethiopia & Specimen & Assefa A et al. \\
\hline 266. & & 1. linteus & Teng 3279 & JX985738 & Xylosoma & China & Specimen & Assefa A et al. \\
\hline 267. & S. sp. 2 & P. sp. & DLL 2010-102 & JQ673184 & $\begin{array}{l}\text { Populus } \\
\text { tremuloides }\end{array}$ & USA & Strain & Brazee NJ et al. \\
\hline 268. & & S. vaninii & DLL 2010-102 & KU139197 & $\begin{array}{l}\text { Populus } \\
\text { tremuloides }\end{array}$ & USA & Strain & Brazee NJ \\
\hline 269. & S. sp. 3 & P. baumii & SFC 20001106-4 & AF534066 & & South Korea & Strain & Lim YW et al. \\
\hline 270. & $\begin{array}{l}\text { not } \\
\text { Sanghuangporus }\end{array}$ & S. baumii & DL 101 & KP974834 & & China & Strain & Sun T et al. \\
\hline 271. & $\begin{array}{l}\text { not } \\
\text { Sanghuangporus }\end{array}$ & 1. vaninii & WN-3 & HQ845057 & & China & Strain & Hu W \& Deng X \\
\hline
\end{tabular}

269 ITS sequences (31 newly sequenced and 238 downloaded from GenBank) from Sanghuangporus species was used to construct a preliminary phylogenetic framework for this genus. An alignment of 941 characters resulted from this dataset, and HKY + G was estimated as the bestfit evolutionary model for phylogenetic analysis. The ML search stopped after 850 bootstrap replicates. All chains in BI converged after ten million generations, which is indicated by the estimated sample sizes (ESSs) of all parameters above 500 and the potential scale reduction factors (PSRFs) close to 1.000. The ML and BI algorithms generated nearly congruent topologies in the main lineages (Additional file 1: Tree S1, Additional file 2: Tree S2). Therefore, only the topology from the ML algorithm is visualized in a circle form here; the midpoint-rooted tree recovered 13 species and four undescribed lineages of
Sanghuangporus (Fig. 1). The one species gap compared with the 14 accepted species is a result of collections previously identified as $S$. quercicola and S. toxicodendri (this species is represented by collections Wu 1805-2, Wu 1805-3, Wu 1805-5, Wu 1807-2, Wu 1807-3 and Wu 1807-4) nesting within a single clade (Fig. 1). Of the 13 recovered species of Sanghuangporus, the clades of S. lonicericola and S. sanghuang did not receive good statistical support, the clade of $S$. alpinus was strongly supported just by the $\mathrm{BI}$ algorithm, and the other species were all strongly supported by both the ML and the BI algorithms (Additional file 1: Tree S1, Additional file 2: Tree S2). Sanghuangporus microcystideus merged with $S$. sp. 1 in the tree inferred from the ML algorithm (Fig. 1, Additional file 1: Tree S1), but was separated from S. sp. 1 in the BI tree (Additional file 2: Tree S2). The relationship between $S$. 


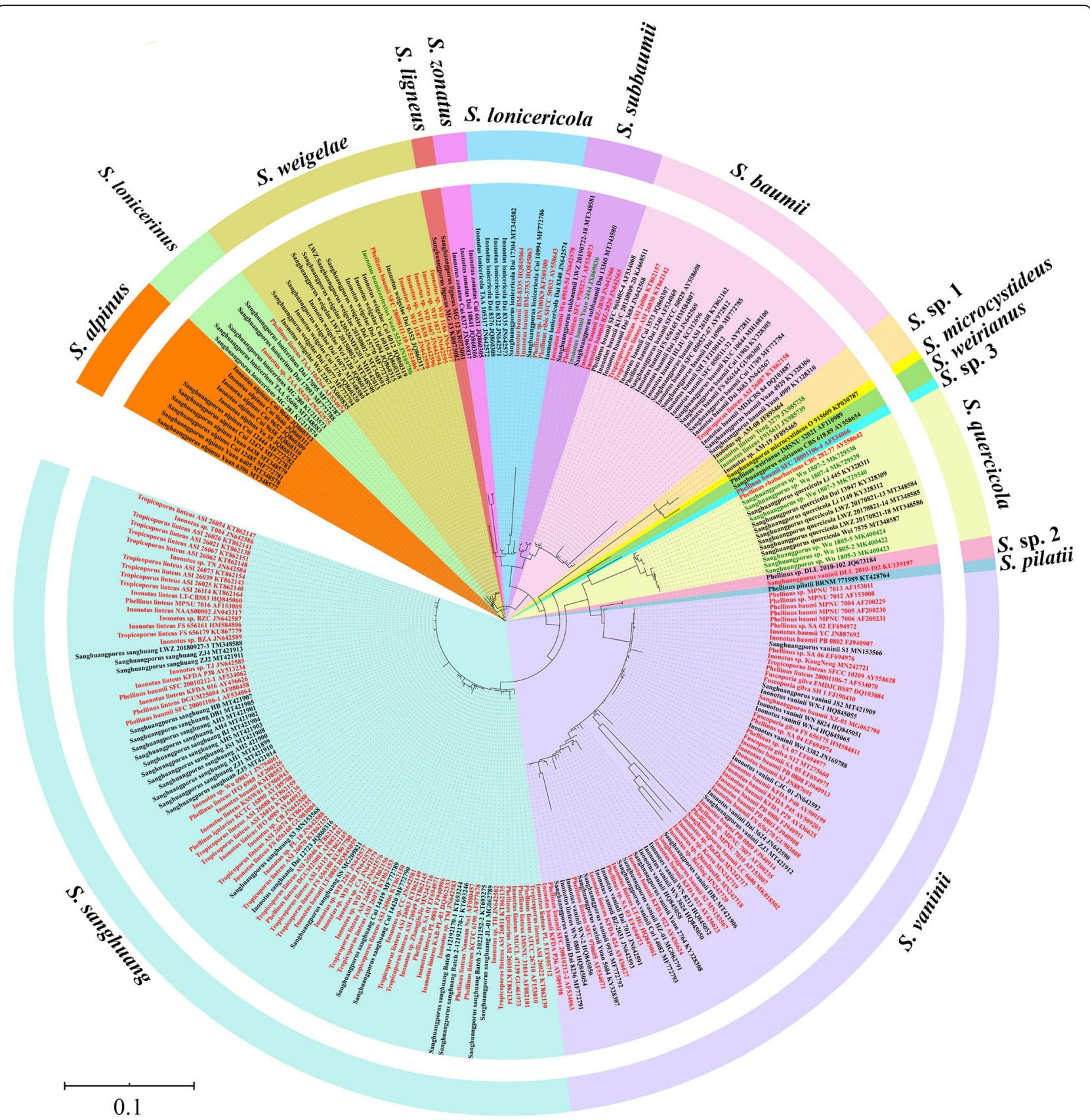

Fig. 1 The phylogenetic tree inferred from 269 ITS sequences. The topology was generated from the maximum likelihood algorithm. The tips in green represent mislabeled specimens, while those in red represent mislabeled strains

microcystideus and S. sp. 1 is still not clear, so we tentatively treat the specimen O 915609 as the single representative of $S$. microcystideus. One undescribed lineage including seven collections BZ-2029, BZ-2030, Dai 13360, LWZ 20190722-18, SFC 970527-1, Wu 0910-54 and Yuan 2444 showed a close relationship with S. baumii (Fig. 1).

In GenBank, species names from 10 out of 77 phylogenetically analyzed specimens were misapplied (tips labeled in green in Fig. 1), while those from 134 out of 192 phylogenetically analyzed strains were wrongly identified to species level (tips labeled in red in Fig. 1). Furthermore, two ITS sequences (HQ845057 and KP974834) of strains labeled as species of Sanghuangporus were extremely deviant and did not belong to the genus (Table 1). Most of these errors came from submissions by non-taxonomists. Therefore, to circumscribe species in Sanghuangporus, we selected the ITS sequences submitted to GenBank by 
taxonomists for a new round of phylogenetic analysis (Table 1). The new dataset included 122 ITS sequences and resulted in an alignment of 871 characters with $\mathrm{HKY}+\mathrm{I}+\mathrm{G}$ as the best-fit evolutionary model. The ML search stopped after 450 bootstrap replicates. All chains in BI converged after four million generations, which is indicated by the ESSs of all parameters above 1000 and the PSRFs close to 1.000 . The ML and BI algorithms generated nearly congruent topologies in the main lineages, and so only the midpoint-rooted ML tree is presented along with the BPPs at the nodes (Fig. 2). As in Fig. 1, this tree also recovered 13 species of Sanghuangporus with $S$. quercicola and S. toxicodendri nested within a single clade (Fig. 2). Among these 13 species, the clade of $S$. lonicericola was still not strongly supported, and the clades of $S$. alpinus and $S$. sanghuang were moderately supported from the ML algorithm and fully supported from the BI algorithm, while the clades of all other species received strong statistical support from both the ML and the BI algorithms (Fig. 2). Moreover, in the seven collections of the undescribed lineage close to $S$. baumii in Fig. 1, four were sampled in the new dataset, and the independence of these four collections and their affinity to S. baumii were also strongly supported (Fig. 2). Therefore, this undescribed lineage is described as a new species, $S$. subbaumii, below.

Molecular species delimitation was estimated on the tree generated from the new dataset with 122 selected ITS sequences. The MPTP method supported the independence of 11 species, while Sanghuangporus alpinus, S. lonicerinus and S. weigelae were recovered as a single species (Additional file 3: Fig. S1).

To further explore the species relationships among Sanghuangporus, the alignment with 122 selected ITS sequences underwent a genetic distance analysis. The ranges of the within and between species genetic distances are mostly non-overlapping (Additional file 4: Table S1). Sanghuangporus microcystideus and S. pilatii, each represented by a single collection, were excluded from the within species analysis. Regarding other species of Sanghuangporus, the genetic distances within $S$. vaninii, S. weirianus and S. zonatus were $0-1.72 \%, 2.68 \%$ and $0-1.71 \%$, respectively, whereas those within other species were no more than $1.30 \%$ and as low as $0.00 \%$ within S. ligneus (Additional file 4: Table S1). Regarding the genetic distances between species, all were above $1.30 \%$ except that those between $S$. alpinus and $S$. lonicerinus, and S. baumii and S. subbaumii were 1.03$2.86 \%$ and $1.19-3.07 \%$, respectively. Across all pairwise comparisons between species, most (84 of 91) had distances above the maximum within species distance of
2.68\% (Additional file 4: Table S1). Furthermore, distances between $S$. microcystideus and all other species were more than $8.90 \%$ and those between S. pilatii and all other species were more than $2.69 \%$ (Additional file 4 : Table S1).

Based on an integrative taxonomic approach, 14 species of Sanghuangporus are accepted here. Their taxonomic information and reliable ITS sequences (from holotypes where possible) are provided below. Regarding S. baumii, S. lonicericola, S. lonicerinus, S. microcystideus, S. pilatii, S. vaninii, and $S$. weirianus, their holotypes were too old (50 years old or more) and so were unlikely to be successfully sequenced. Moreover, certain institutions did not make holotypes available for sequencing. Therefore, we use ITS sequences from other reference collections as reliable ITS sequences for those species.

Fifty-four ITS sequences of $S$. baumii, S. sanghuang and $S$. vaninii, the most common species in medicinal studies and products (Zhou et al. 2020), were further retrieved from the dataset with 122 selected sequences. These 54 sequences were realigned and the alignment is presented with shaded background (Additional file 5: Fig. S2). From this alignment, ten potential diagnostic sequences with two to six nucleotide differences were identified for HRCA to differentiate species: two for $S$. baumii, two for $S$. sanghuang and six for $S$. vaninii (Additional file 5: Fig. S2, Table 2).

\section{TAXONOMY}

Sanghuangporus alpinus (Y.C. Dai \& X.M. Tian) L.W. Zhou \& Y.C. Dai, Fungal Diversity 77: 340 (2016).

Basionym: Inonotus alpinus Y.C. Dai \& X.M. Tian, Fungal Diversity 58: 162 (2013).

Type: China: Tibet: Linzhi County, Lulang, on living angiosperm tree, 24 Sept. 2010, B.K. Cui, Cui 9658 (BJFC - holotype).

ITS barcoding sequence: JQ860310 (from holotype).

Sanghuangporus baumii (Pilát) L.W. Zhou \& Y.C. Dai, Fungal Diversity 77: 340 (2016).

Basionym: Phellinus baumii Pilát, Bull. trimest. Soc. mycol. Fr. 48: 25 (1932).

Synonym: Inonotus baumii (Pilát) T. Wagner \& $\mathrm{M}$. Fisch., Mycologia 94: 1009 (2002).

Type: Russia: Primorsky Krai: Vladivostok, on trunk of Syringae, 5 June 1928, M.K. Ziling 267 (PRM 189012 holotype).

Reference collection: China: Heilongjiang: Yichun, Fenglin nature reserve, on living trunk of Syringa, 8 Sept. 2002, Y.C. Dai, Dai 3683 (IFP) 


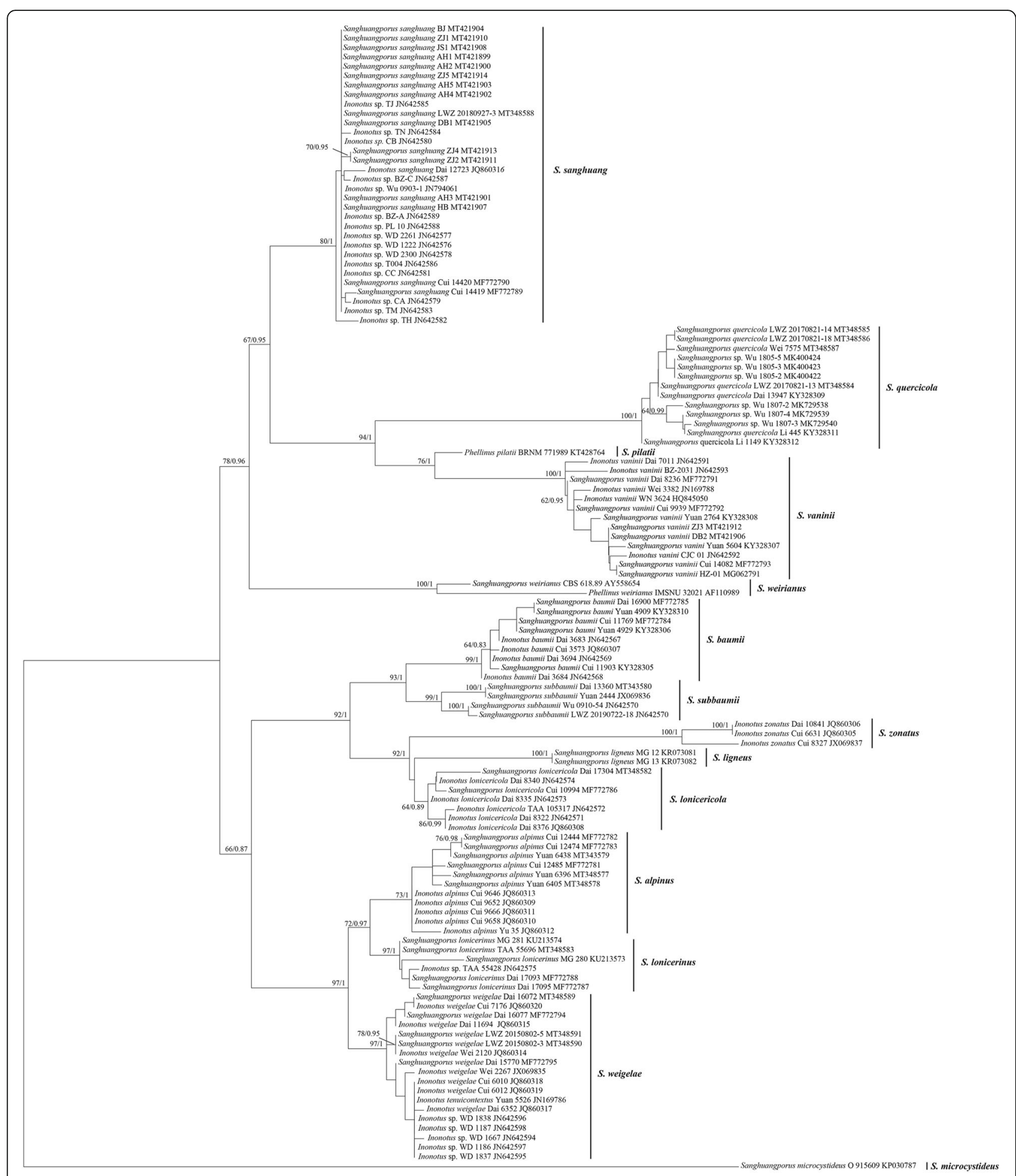

$\stackrel{0.005}{1}$

Fig. 2 The phylogenetic tree inferred from ITS sequences submitted by taxonomists. The topology was generated from the maximum likelihood algorithm, and bootstrap values and Bayesian posterior probabilities simultaneously above $50 \%$ and 0.8 , respectively, are presented at the nodes 
Table 2 Diagnostic sequences with potential for discriminating Sanghuangporus baumii, S. sanghuang, and S. vaninii using Hyperbranched Rolling Circle Amplification. Label and position in alignment are as in Additional file 5: Fig. S2

\begin{tabular}{lllll}
\hline Label & Differentiated species & Diagnostic sequence & Position in alignment & Number of diagnostic nucleotides \\
\hline A & S. sanghuang & AWYTY & $41-45$ & 5 \\
B & S. vaninii & TCA & $85-87$ & 3 \\
C & S. vaninii & CTG & $143-145$ & 3 \\
D & S. baumii & CGGTAGGAA & $159-167$ & 4 \\
E & S. vaninii & GAGCGG & $219-224$ & 6 \\
F & S. vaninii & CCCCC & $264-278$ & 4 \\
G & S. vaninii & AG & $556-557$ & 2 \\
H & S. baumii & AGG & $650-652$ & 2 \\
I & S. vaninii & ACG & $664-666$ & 2 \\
J & S. sanghuang & TT & $690-691$ & 2 \\
\hline
\end{tabular}

ITS barcoding sequence: JN642567 (from the reference collection cited above, proposed by Zhou et al. (2020) and accepted here).

Sanghuangporus ligneus Ghob.-Nejh., Mycol. Progr. 14(90): 2 (2015).

Type: Iran: East Azerbaijan: Khoda-Afarin, Kalaleh-Eslami, Darana, deciduous forest with Quercus macranthera, Lonicera, Cornus mas, and Crataegus, on stem of living Lonicera caucasica, 10 May 2008, M. Ghobad-Nejhad, Ghobad-Nejhad 1152 (ICH - holotype).

ITS barcoding sequence: KR073081 (from holotype).

Sanghuangporus lonicericola (Parmasto) L.W. Zhou \& Y.C. Dai, Fungal Diversity 77: 340 (2016).

Basionym: Phellinus lonicericola Parmasto, Folia cryptog. Estonica 38: 59 (2001).

Synonym: Inonotus lonicericola (Parmasto) Y.C. Dai, Fungal Diversity 45: 276 (2010).

Type: Russia: Primorsky Krai: Lazovsky Nature Reserve, Petrov island, on trunk of Lonicera ruprechtiana in Taxus mixed forest, 2 Sept. 1961, E. Parmasto (TAA-M 013933 - holotype).

Reference collection: China: Heilongjiang: Ningan County, Jingpohu National Scenic Area, on living trunk of Lonicera, 8 Sept. 2007, Y.C. Dai, Dai 8376 (IFP)

ITS barcoding sequence: JQ860308 (from the reference collection cited above, proposed by Zhou et al. (2020) and accepted here).

Sanghuangporus lonicerinus (Bondartsev) Sheng $\mathrm{H}$. Wu et al., Fungal Diversity 77: 340 (2016).

Basionym: Fomes lonicerinus Bondartsev, Acta Inst. Bot. Acad. Sci. USSR Plant. Crypt., Ser. II: no. 500 (1935).

Synonyms: Phellinus lonicerinus (Bondartsev) Bondartsev \& Singer, Annls mycol. 39: 56 (1941).
Cryptoderma lonicerinum (Bondartsev) Imazeki, Bull. Tokyo Sci. Mus. 6: 107 (1943).

Porodaedalea lonicerina (Bondartsev) Imazeki, Col. Ill. Mushrooms Japan, 2: 191 (1989).

Inonotus lonicerinus (Bondartsev) Sheng $\mathrm{H}$. Wu et al., Bot. Studies (Taipei) 53: 140 (2012).

Type: Uzbekistan: Samarkand: Sarymat, on trunk of Lonicera tatarica, 1926, E. Czerniakowsk (LE 22512 lectotype designated by Bondartsev 1953).

Reference collection: Turkmenistan: Bakharden: Bakharden, Arvaz, Montes Kopet-dagh, on Lonicera, 17 Oct. 1971, E. Parmasto (TAA 55428)

ITS barcoding sequence: JN642575 (from the reference collection cited above, proposed by Zhou et al. (2020) and accepted here).

Sanghuangporus microcystideus (Har. \& Pat.) L.W. Zhou \& Y.C. Dai, Fungal Diversity 77: 340 (2016).

Basionym: Phellinus microcystideus Har. \& Pat., Bull. Mus. natn. Hist. nat., Paris 15: 90 (1909).

Synonym: Fomes microcystideus (Har. \& Pat.) Sacc. \& Trotter, Syll. Fung. 21: 286 (1912).

Type: Congo: Moyen Oubangui: Grande Forêt, M.A. Chevalier 11431 (FH - holotype).

Reference collection: Tanzania: Arusha: Arusha National Park, Mount Meru, on trunk of Olea africana, 18 Feb. 1976, R. Harjula (O 915609)

ITS barcoding sequence: KP030787 (from the reference collection cited above, proposed by Zhou et al. (2020) and accepted here).

Sanghuangporus pilatii (Černý) Tomšovský, Phytotaxa 239: 84 (2015).

Basionym: Phellinus pilatii Černý, Česká Mykol. 22(1): 2 (1968).

Synonym: Porodaedalea pilatii (Černý) Fiasson \& Niemelä, Karstenia 24(1): 26 (1984). 
Type: Czech Republic: Břeclav: Tvrdonice, 8 Oct. 1955, A. Černý (PRM 628393 - holotype).

Reference collection: Czech Republic: Břeclav: Nové Mlýny, Křivé jezero National Nature Reserve, on Populus alba, 22 Oct. 2011, M. Tomšovský 41/2011 (BRNM 771989)

ITS barcoding sequence: KT428764 (from the reference collection cited above, proposed by Zhou et al. (2020) and accepted here).

Sanghuangporus quercicola Lin Zhu \& B.K. Cui, Phytotaxa 311: 271 (2017).

Synonym: Sanghuangporus toxicodendri Sheng H. Wu et al., MycoKeys 57: 106 (2019).

Type: China: Henan: Neixiang County, Baotianman Nature Reserve, on dead tree of Quercus, 25 Aug. 2006, J. Li, Li 1149 (BJFC - holotype).

ITS barcoding sequence: KY328312 (from holotype).

Sanghuangporus sanghuang (Sheng $\mathrm{H}$. Wu et al.) Sheng H. Wu et al., Fungal Diversity 77: 340 (2016).

Basionym: Inonotus sanghuang Sheng H. Wu et al., Bot. Studies (Taipei) 53: 140 (2012).

Type: China: Jilin: Baishan City, on Morus sp., Mar. 2009, S.H. Wu, Wu 0903-1 (TNM - holotype).

ITS barcoding sequence: JN794061 (from holotype).

Sanghuangporus subbaumii Shan Shen, Y.C. Dai \& L.W. Zhou, sp. nov. (Figs. 3 and 4).

MycoBank MB838235.

Etymology: subbaumii (Lat.), refers to the similarity to Sanghuangporus baumii.

Diagnosis: Differing from S. baumii in having resupinate, effused-reflexed to pileate basidiomes, acute pileal margin and longer hymenial setae ( $>20 \mu \mathrm{m}$ in length).

Type: China: Shanxi: Jiaocheng County, Pangquangou Nature Reserve, on fallen trunk of Prunus sp., 10 Aug. 2013, Y.C. Dai, Dai 13360 (BJFC - holotype; HMAS 281653 - isotype).

Description: Basidiomes perennial, resupinate, effused-reflexed to pileate, without odor or taste and hard corky when fresh, woody hard when dry; to $20 \mathrm{~cm}$ long and $5 \mathrm{~cm}$ wide when resupinate. Pilei dimidiate, ungulate in section, projecting to $3.5 \mathrm{~cm}$ wide, $6 \mathrm{~cm}$ long and $4 \mathrm{~cm}$ thick at base. Pileal surface dark brown and velutinate when juvenile, mousegrey to black, glabrous and cracked with age, concentrically zonate and narrowly sulcate; margin yellow brown, acute. Pore surface yellowish brown, glancing; sterile margin distinct, yellowish; pores angular to circular, 5-7 per mm; dissepiments thin, entire. Context yellowish brown to dark brown, woody hard, to $3.5 \mathrm{~cm}$ thick. Tubes yellowish brown, darker than pore surface, woody hard, to $0.5 \mathrm{~cm}$ long. Hyphal system monomitic in context, dimitic in trama; generative hyphae simple septate; tissue darkening but

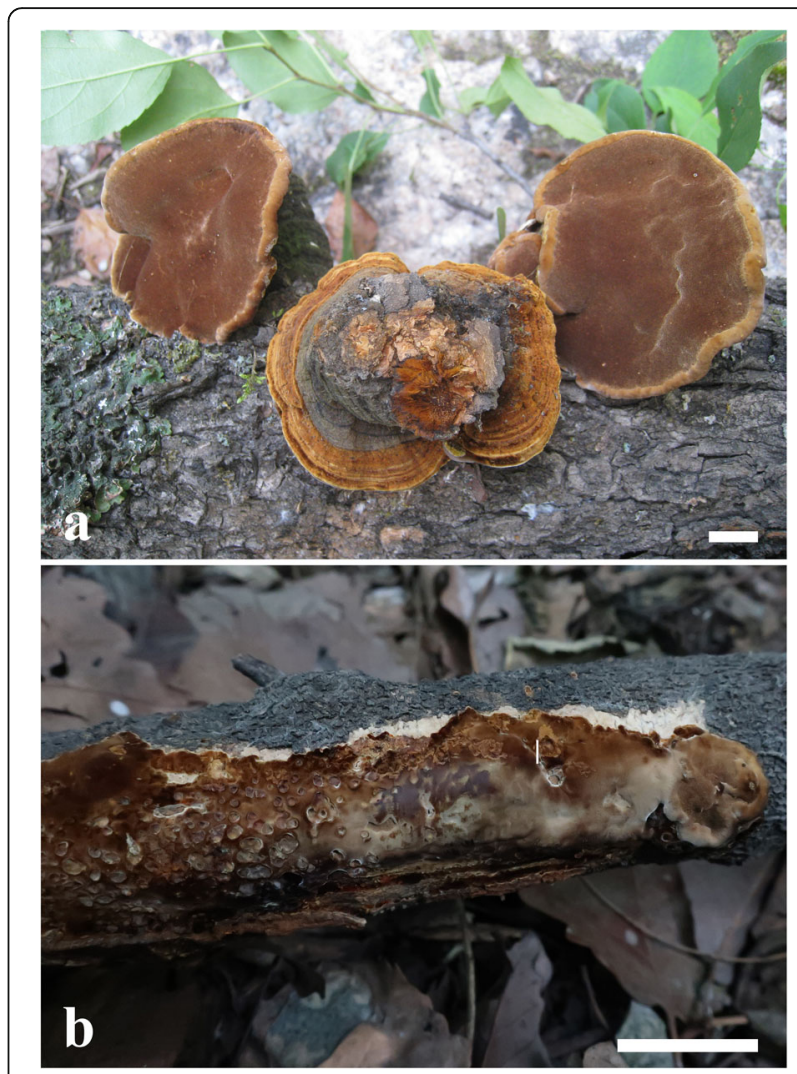

Fig. 3 Basidiomes of Sanghuangporus subbaumii in situ. a Dai 13360 (holotype). b LWZ 20190722-18 (paratype). Bars: 2 cm

otherwise unchanged in $\mathrm{KOH}$. Context generative hyphae occasionally slightly thick-walled with a wide lumen and yellowish, mostly thick-walled with a narrow lumen and yellowish brown, unbranched, frequently septate, more or less regularly arranged, 3.5-4 $\mu \mathrm{m}$ diam. Tubes generative hyphae thin to slightly thick-walled, hyaline, occasionally branched, frequently septate, 3-4.5 $\mu \mathrm{m}$ diam; skeletal hyphae dominant, thick-walled with a narrow lumen, yellowish brown, unbranched, rarely septate, subparallel along the tubes, $2.2-3.7 \mu \mathrm{m}$ diam. Hymenial setae frequent in the mature hymenium, subulate to ventricose, dark brown, thick-walled, 20-35 $\times 7-12 \mu \mathrm{m}$. Cystidioles subulate, with narrow and tapering apex, hyaline, 15-20 $\times$ 4-6 $\mu \mathrm{m}$. Basidia barrel-shaped to broadly clavate, with four sterigmata and a simple septum at the base, hyaline, $20-25 \times 7-9 \mu \mathrm{m}$; basidioles in shape similar to basidia, but slightly smaller. Basidiospores broadly ellipsoid to subglobose, yellowish, slightly thick-walled, smooth, non-amyloid, non-dextrinoid, moderately cyanophilous, $(3.8-) 4-4.9(-5.2) \times 3.1-3.8(-3.9) \quad \mu \mathrm{m}, \mathrm{L}=4.35 \mu \mathrm{m}, \mathrm{W}=$ $3.41 \mu \mathrm{m}, \mathrm{Q}=1.24-1.31(n=60 / 2)$.

Notes: Sanghuangporus subbaumii mostly resembles $S$. baumii, but the latter species differs in having pileate basidiomes always, obtuse pileal margin and shorter hymenial setae $(<20 \mu \mathrm{m}$ in length; Dai 2010). The 


\section{0}

$\mathbf{a}$

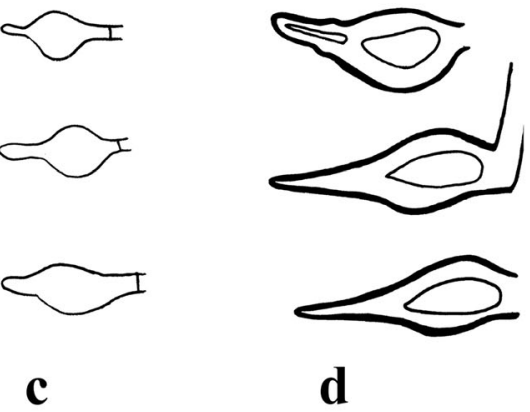

b
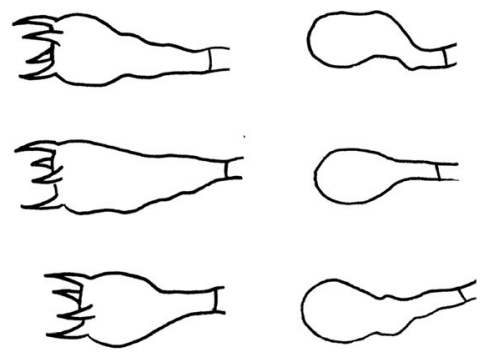

C

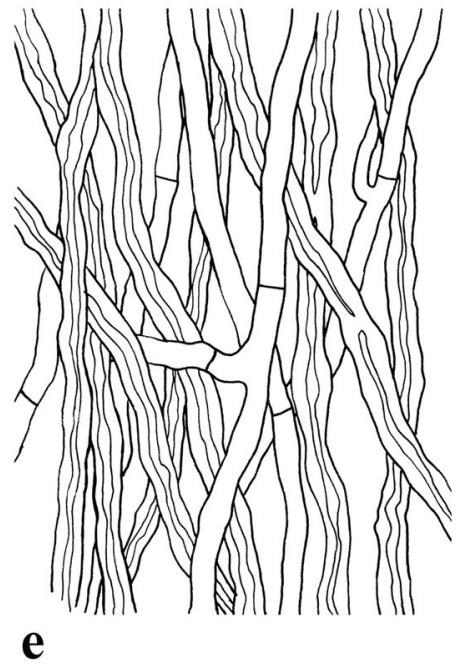

f

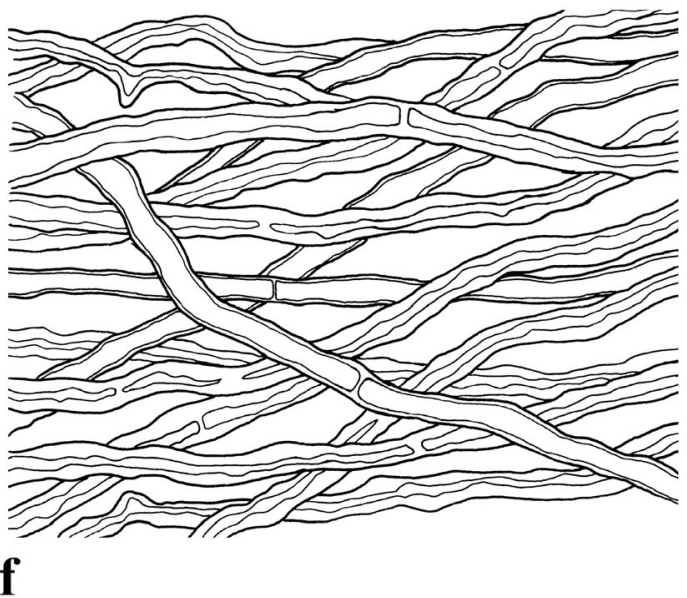

Fig. 4 Microscopic structures of Sanghuangporus subbaumii (drawn from Dai 13360, holotype). a Basidiospores. b basidia and basidioles. c cystidioles. $\mathbf{d}$ hymenial setae. e hyphae from trama. $\mathbf{f}$ hyphae from context. Bars: $\mathrm{a}=5 \mu \mathrm{m}, \mathrm{b}-\mathrm{e}=10 \mu \mathrm{m}$

resupinate to pileate basidiomes make $S$. subbaumii similar to $S$. vaninii, but $S$. vaninii lacks cystidioles and has a thin black zone separating heterogeneous context (Dai 2010).

ITS barcoding sequence: MT348580 (from holotype).

Additional specimen examined: China: Beijing: Shangfangshan Forest Park, on fallen angiosperm trunk, 22 July 2019, L.W. Zhou, LWZ 20190722-18 (HMAS 281654).

Sanghuangporus vaninii (Ljub.) L.W. Zhou \& Y.C. Dai, Fungal Diversity 77: 340 (2016).

Basionym: Phellinus vaninii Ljub., Bot. Mater. 15: 115 (1962).

Synonym: Inonotus vaninii (Ljub.) T. Wagner \& $\mathrm{M}$. Fisch., Mycologia 94: 1009 (2002).

Type: Russia: Primorsky Krai: Shkotovsky District, watershed of the Maykhe river, Maykhinsky forestry, Verkhne-Maykhinskaya forest area, Peyshula, quarter 119, in valley of pine-broadleaved forest, on dried aspen tree, 14 Aug. 1951, L.V. Lyubarskiy (LE 22523 - holotype).

Reference collection: China: Jilin: Antu County, Changbaishan, on fallen trunk of Populus davidiana, 26 Aug. 2005, Y.C. Dai, Dai 7011 (IFP)

ITS barcoding sequence: JN642591 (from the reference collection cited above, proposed by Zhou et al. (2020) and accepted here).

Sanghuangporus weigelae (T. Hatt. \& Sheng H. Wu) Sheng H. Wu et al., Fungal Diversity 77: 340 (2016).

Basionym: Inonotus weigelae T. Hatt. \& Sheng $\mathrm{H}$. Wu, Bot. Studies (Taipei) 53: 143 (2012).

Synonym: Inonotus tenuicontextus L.W. Zhou \& W.M. Qin, Mycol. Progr. 11: 793 (2012).

Type: Japan: Nagano: Chino, Minoto, on Weigela coraeensis, 19 Sept. 1993, T. Hattori, F16899 (TFM - holotype).

ITS barcoding sequence: JN642596 (from holotype). 
Sanghuangporus weirianus (Bres.) L.W. Zhou \& Y.C. Dai, Fungal Diversity 77: 340 (2016).

Basionym: Fomes weirianus Bres., Stud. Trent., Classe II, Sci. Nat. Econ. 7(1): 5 (1926).

Synonyms: Phellinus weirianus (Bres.) Gilb., J. Ariz. Acad. Sci. 7: 137 (1972).

Inonotus weirianus (Bres.) T. Wagner \& M. Fisch., Mycologia 94: 1009 (2002).

Type: USA: New Mexico: on trunk of Juglans rupestris, 25 Oct. 1911, G.G. Hedgcock \& W.H. Long (BPI 235278 - holotype).

Reference collection: USA: Arizona: on Juglans major, 27 Aug. 1967, R.L. Gilbertson 6975-S (IMSNU 32021)

ITS barcoding sequence: AF110989 (from the reference collection cited above, proposed by Zhou et al. (2020) and accepted here).

Sanghuangporus zonatus (Y.C. Dai \& X.M. Tian) L.W. Zhou \& Y.C. Dai, Fungal Diversity 77: 341 (2016).

Basionym: Inonotus zonatus Y.C. Dai \& X.M. Tian, Fungal Diversity 58: 165 (2013).

Type: China: Hainan: Jianfengling Nature Reserve, on living angiosperm tree, 11 May 2009, B.K. Cui, Cui 6631 (BJFC - holotype).

ITS barcoding sequence: JQ860305 (from holotype).

\section{DISCUSSION}

In this study, we summarized all available ITS barcoding sequences bearing the name "Sanghuang" in GenBank. A total of 271 ITS sequences related to "Sanghuang", including 31 newly generated sequences from this study, were analyzed. In association with previous information of morphology, hosts, and multilocus-based phylogeny, 14 species are accepted as members of Sanghuangporus including the new species $S$. subbaumii described herein. We also synonymize $S$. toxicodendri under S. quercicola.

Sanghuangporus subbaumii has a phylogenetically close relationship to S. baumii; however, these two species form two distinct lineages with strong support (Additional file 1: Tree S1, Additional file 2: Tree S2, Fig. 2). Moreover, S. subbaumii and S. baumii were also estimated as two independent species using the MPTP method (Additional file 3: Fig. S1), and for ITS the interspecific distance is $1.19-3.07 \%$, generally above the cutoff value of interspecific distances (1.30\%) within Sanghuangporus (Additional file 4: Table S1). Besides molecular evidence, morphological differences between these two species are also clear. Geographically, $S$. subbaumii is only known from North China, whereas Chinese collections of $S$. baumii are distributed in north-east China (Table 1).

Sanghuangporus toxicodendri was recently described from specimens collected from Toxicodendron sp. in Hubei, central China (Wu et al. 2019b) and resembles $S$. quercicola, another species originally described from central China (Zhu et al. 2017). However, in the publication introducing S. toxicodendri (Wu et al. 2019b) the separation from $S$. quercicola was not well-supported phylogenetically. Moreover, the morphological differences between these two species are slight (such as for basidiospore length) or involve variable characters that do not have taxonomic signal (such as the surface color of the pileal margin) (Zhu et al. 2017; Wu et al. 2019b). In the current phylogenetic analyses, the six specimens of $S$. toxicodendri, three specimens of S. quercicola and four additional collections merged in a fully supported clade (Additional file 1: Tree S1, Additional file 2: Tree S2, Fig. 2). The mPTP-based estimation of species delimitation also treated S. toxicodendri and S. quercicola as a single species (Additional file 3: Fig. S1) and the intraspecific distances among ITS sequences under both names were $0-1.11 \%$, well below the threshold of $1.30 \%$ (Additional file 4: Table S1). Therefore, S. toxicodendri and $S$. quercicola are considered conspecific, and $S$. quercicola has priority by publication date over $S$. toxicodendri.

The clade of S. lonicericola was present but not wellsupported in our phylogenetic analyses (Additional file 1: Tree S1, Additional file 2: Tree S2, Fig. 2). Similarly, the clades of $S$. alpinus and $S$. sanghuang were not strongly supported by the ML algorithm (Fig. 2). For $S$. lonicericola and S. alpinus, despite the lack of support in one or both analyses, each formed a distinct clade, and for both species distances to other species were above the threshold of $1.30 \%$ (S. lonicericola minimum $2.19 \%$ and S. sanghuang minimum 2.90\%; Additional file 4: Table S1). In addition, S. alpinus, S. lonicerinus, and S. weigelae, even though forming three independent lineages, were considered conspecific by the MPTP method (Additional file 3: Fig. S1). However, the interspecific distances for ITS between $S$. weigelae and each of $S$. alpinus and S. lonicerinus are above the cut-off value of interspecific distances (1.30\%) within Sanghuangporus (Additional file 4: Table S1). Regarding the pair of $S$. alpinus and S. lonicerinus, for ITS the between species distance $(1.03-2.86 \%)$ was generally above the intraspecific distances within either species $(0-1.08 \%$ and 0 $1.18 \%$, respectively; Additional file 4: Table S1). Moreover, the monophyly of $S$. alpinus was strongly supported by the BI algorithm and that of S. lonicerinus was strongly supported by both the ML and the BI algorithms (Fig. 2). Besides, morphological delimitations among these five species are stable (Wu et al. 2012a; Tian et al. 2013; Zhou et al. 2016). Taking all this into account, we accept $S$. alpinus, S. lonicericola, S. lonicerinus, S. sanghuang, and S. weigelae as five independent species.

Sanghuangporus vaninii, S. weirianus, and S. zonatus are the only three species with intraspecific ITS 
distances of more than $1.30 \%(0-1.72 \%, 2.68 \%$ and $0-$ $1.71 \%$, respectively; Additional file 4: Table S1). However, they all received strong support as independent species (Additional file 1: Tree S1, Additional file 2: Tree S2, Fig. 2, Additional file 3: Fig. S1). As one of the most commonly cultivated species, several cultivars of $S$. vaninii were included in the evaluation of genetic distances of ITS sequences (Zhou et al. 2020; Table 1). The procedure of cultivation with continuous passage culture can dramatically accelerate the accumulation of genetic variation, which may result in the higher intraspecific ITS difference in $S$. vaninii. Noteworthily, branch lengths of the only two available collections of $S$. weirianus were markedly different even though the two strains were from the same original isolate (Fig. 2). Regarding $S$. zonatus, two collections from Hainan, South China grouped together with full statistical support, and then formed a fully supported clade with a collection from Yunnan, Southwest China (Table 1, Figs. 1 and 2). Both $S$. weirianus and $S$. zonatus are poorly collected species, and a more comprehensive sampling of these two species in phylogenetic analyses will further clarify their intraspecific relationships. For now, we tentatively accept them as monophyletic species.

A study by Nilsson et al. (2006) revealed that about $10-21 \%$ of 51,000 fungal ITS sequences available at that time in the International Nucleotide Sequence Databases were annotated with incorrect taxonomic information. More recently, this proportion has increased to almost 30\% (Hofstetter et al. 2019). Regarding "Sanghuang", more than half (or say 146) of the ITS sequences labeled as such, were found to be mislabeled, implying that the proportion of incorrectly labeled ITS sequences for "Sanghuang" is much higher than the average proportion for all fungal groups. This phenomenon may be attributable to the medicinal properties of "Sanghuang", which attracts much more attention from non-taxonomists who submit ITS sequences to GenBank. Consequently, the numerous errors result in chaos with BLAST searches, especially for non-taxonomists. Although the RefSeq Targeted Loci (RTL) database has been initiated for fungal ITS sequences from type collections (Schoch et al. 2014), only two species of Sanghuangporus, viz. S. alpinus and S. zonatus were reannotated and deposited under accession numbers of NR_158887 and NR 166366. Actually, ITS sequences from six holotypes of accepted Sanghuangporus species are available in GenBank. This number increases to eight, if two synonyms of other species of Sanghuangporus, viz. Inonotus tenuicontextus and $S$. toxicodendri are considered. In UNITE (Nilsson et al. 2019), tens of species hypotheses belonging to Sanghuangporus are available under various threshold values at species level; however, not all accepted species of Sanghuangporus (such as S. ligneus,
S. pilatii, and S. quercicola) are referred to and the reference sequences for some species hypotheses are not always those from holotypes. Moreover, both RTL and UNITE are not familiar to mycologists working on medicinal studies and government officers in charge of the policy of medicinal fungi, who normally take the first hit of a BLAST search in GenBank as the species name. Therefore, the accuracy of ITS sequences of "Sanghuang" in GenBank is crucial for medicinal studies and commercial development of this fungal genus.

Compared with specimens, many more mislabeled ITS sequences of Sanghuangporus came from cultured strains, and most of those sequences were submitted by nontaxonomists. A typical case is the recent paper on genome sequencing of "Sanghuang" that also submitted six ITS sequences to GenBank (Shao et al. 2020). In GenBank, all these six sequences were labeled as Inonotus sp. rather than species of Sanghuangporus (MN242716-MN242721), while the six strains generating these sequences were named as S. sanghuang (Shao et al. 2020). However, five of the six strains, including the one (labeled as KangNeng) subjected to genome sequencing, are actually $S$. vaninii (Fig. 1, Zhou et al. 2020); i.e. five out of six strains were wrongly identified to species level. Therefore, this species misidentification means that the whole genome sequence of "Sanghuang" may be misapplied in future studies. Shao et al. (2020) also stated that these six strains are commercially cultivated, which further results in the name chaos for commercial products of "Sanghuang". Another publication on genome sequencing identified the genome sequenced strain S12 as Phellinus gilvus according to ITS barcoding region (Huo et al. 2020). However, the corresponding ITS sequence (MT275660) annotated as Fuscoporia gilva in GenBank represents S. vaninii (Fig. 1, Zhou et al. 2020). Another case is a paper devoted to the species identity of "Sanghuang" strains (Han et al. 2016). Thirty strains deposited in the Agricultural Sciences Institute culture collection (Mushroom Research Division, Rural Development Administration, Republic of Korea) were correctly identified as S. vaninii and S. sanghuang according to an ITS-based phylogenetic analysis; however, unfortunately, most of these ITS sequences were mislabeled when being submitted to GenBank.

Ten mislabeled ITS sequences found in the current study came from basidiomes. These errors were caused mainly by taxonomic revisions of certain species. Six sequences of specimens Wu 1805-2, Wu 1805-3, Wu 1805-5, Wu 1807-2, Wu 1807-3 and Wu 1807-4 that were originally labeled as Sanghuangporus sp. but later cited under S. toxicodendri by Wu et al. (2019b) are accepted to represent S. quercicola. Yuan 2444, previously considered as $S$. baumii, was nested within the lineage segregated from S. baumii as a new species S. subbaumii (Figs. 1 and 2, Additional file 3: Fig. S1). Consequently, 
the ITS sequence of Yuan 2444 (JX069836) is corrected to $S$. subbaumii (Table 1). Another mislabeled sequence was generated from a specimen originally described as Inonotus tenuicontextus (Zhou and Qin 2012). Although this species was published online earlier than Inonotus weigelae (basionym of S. weigelae; Wu et al. 2012a; Tian et al. 2013), its online date is before 1 January 2012 and thus the name was not effectively published online according to Art. 29.1 of the ICNafp (Turland et al. 2018). Inonotus tenuicontextus was then treated as a later synonym of I. weigelae (Tian et al. 2013). Therefore, this mislabeled sequence is accepted to represent $S$. weigelae (Table 1).

Although intact mature basidiomes of "Sanghuang" are not difficult to identify to species level morphologically and in a short time by taxonomists working on this group, most of the commercial products are small pieces or even powders. Normally, it is impossible to rapidly determine which species those commercial products represent. As for other traditional medicinal mushrooms (Raja et al. 2017), species names of Sanghuangporus are sometimes misapplied to certain products of "Sanghuang" (Shao et al. 2020). This confused situation to some extent restricts the commercial development of "Sanghuang" (Zhou 2020). Therefore, to standardize the "Sanghuang" industry, ten reference sequences are provided for HRCA based on the accurate boundaries among three commonly studied and cultivated species, viz. S. baumii, S. sanghuang, and S. vaninii (Lin et al. 2017; Zhou et al. 2020). HRCA is an isothermal amplification approach and thus provides a rapid, simple and low-cost detection of specific nucleic acid sequences (Nilsson et al. 1994; Lizardi et al. 1998) even for single nucleotide differences (Nilsson et al. 1997). This approach has been widely used for the clinical detection of human pathogenic microfungi (Zhou et al. 2008; Trilles et al. 2014; Rodrigues et al. 2015) and, recently, was also reported for the rapid detection of poisonous macrofungi (He et al. 2019a, 2019b). Regarding lethal Amanita species, nucleotide differences greater than two allowed species identification using the $\alpha$-amanitin gene ( $\mathrm{He}$ et al. 2019a). Here, for Sanghuangporus a set of candidates for future testing is provided that have diagnostic sequences containing between two and six nucleotide differences.

\section{CONCLUSION}

In order to promote medicinal studies and industrial development, the ITS barcoding region of Sanghuangporus species is here comprehensively analyzed to enable accurate species identification. Firstly, the ITS region is confirmed as an effective barcode in Sanghuangporus. Secondly, the names of all available ITS sequences in GenBank related to "Sanghuang" are carefully revised and where necessary corrected. Thirdly, the intraspecific ITS difference for each species of Sanghuangporus is evaluated to be up to $1.30 \%$ (except S. vaninii, S. weirianus, and $S$. zonatus), while the interspecific ITS difference is above $1.30 \%$ (except between $S$. alpinus and $S$. lonicerinus, and S. baumii and S. subbaumii). This provides a practical cut-off value for BLAST search-based species identification. Finally, ten potential diagnostic sequences are provided for HRCA assay to rapidly differentiate the three commonly studied and cultivated species, viz. S. baumii, S. sanghuang, and S. vaninii. As a follow up, we will suggest reannotation of ITS sequences related to "Sanghuang" to the GenBank administrators, especially to ensure that sequences from holotypes and reference collections for each species of Sanghuangporus are designated as such. Further, we will liaise with UNITE to ensure that appropriate reference sequences are designated for UNITE species hypotheses within Sanghuangporus.

\section{Abbreviations}

BI: Bayesian inference; BPP: Bayesian posterior probability; CB: Cotton Blue; CTAB: Cetyl-trimethyl-ammonium bromide; IKI: Melzer's reagent; ITS: Nuclear ribosomal internal transcribed spacer; $\mathrm{KOH}: 5 \%$ potassium hydroxide; ML: Maximum likelihood; mPTP: Multi-rate Poisson Tree Processes; PCR: Polymerase chain reaction; RTL: RefSeq Targeted Loci

\section{Supplementary Information}

The online version contains supplementary material available at https://doi. org/10.1186/s43008-021-00059-x.

Additional file 1: Tree S1. The phylogenetic tree inferred from 269 ITS sequences. The topology was generated from the maximum likelihood algorithm and bootstrap values are presented at the nodes.

Additional file 2: Tree S2. The phylogenetic tree inferred from 269 ITS sequences. The topology was generated from the Bayesian inference algorithm and Bayesian posterior probabilities are presented at the nodes.

Additional file 3: Figure S1. Molecular species delimitation estimated from the Newick tree file of Fig. 2 using multi-rate Poisson Tree Processes method. The continuous red branches represent a single species.

Additional file 4: Table S1. Genetic distances of ITS sequences between and within species of Sanghuangporus.

Additional file 5: Figure S2. The alignment of Sanghuangporus baumii, S. sanghuang and S. vaninii generated from ITS sequences submitted by taxonomists. Ten potential diagnostic sequences for Hyperbranched Rolling Circle Amplification are labeled in capital letters.

\section{Acknowledgements}

We appreciate Genevieve Gates (Tasmanian Institute of Agriculture, Australia) for help in improving this manuscript, editors and anonymous reviewers for providing constructive comments on the earlier versions of the manuscript, and curators from FH, LE and TAAM for forwarding information of holotypes. Yan Yang and He-Nan Zhang (Institute of Edible Fungi, Shanghai Academy of Agricultural Sciences, China) are thanked for kindly providing cultivated strains for sequencing. Yu-Cheng Dai (BJFC) and Hai-Sheng Yuan (IFP) are thanked for kindly forwarding specimens and strains as loans for sequencing.

Adherence to national and international regulations Not applicable. 


\section{Authors' contributions}

SS, S-LL and L-WZ retrieved and analyzed all data. J-HJ prepared fungal samples and performed molecular sequencing. L-WZ conceived the work and wrote the manuscript. All authors approved the manuscript.

\section{Funding}

The research was financed by the National Natural Science Foundation of China (No. 31970012), Youth Innovation Promotion Association of the Chinese Academy of Sciences (No. 2017240), and Biological Resources Programme, Chinese Academy of Sciences (KFJ-BRP-017-12).

\section{Availability of data and materials}

The materials are available as Additional files 1, 2, 3, 4 and 5. All sequence data generated for this study can be accessed via GenBank: https://www. ncbi.nlm.nih.gov/genbank/. Alignments are available at TreeBase (ID: 26272).

\section{Declarations}

\section{Ethics approval and consent to participate}

Not applicable.

\section{Consent for publication}

Not applicable.

\section{Competing interests}

The authors declare no competing interests

\section{Author details}

${ }^{1}$ State Key Laboratory of Mycology, Institute of Microbiology, Chinese Academy of Sciences, Beijing 100101, China. ${ }^{2}$ University of Chinese Academy of Sciences, Beijing 100049, China. ${ }^{3}$ Institute of Applied Ecology, Chinese Academy of Sciences, Shenyang 110016, China.

Received: 14 August 2020 Accepted: 9 March 2021

Published online: 15 April 2021

\section{References}

Bondartsev AS (1953) Polypores of the European part of the USSR and Caucasus. Academy of Sciences of URSS, Moskva \& Leningrad

Cai C, Ma J, Han C, Jin Y, Zhao G, He X (2019) Extraction and antioxidant activity of total triterpenoids in the mycelium of a medicinal fungus, Sanghuangporus sanghuang. Scientific Reports 9(1):7418. https://doi.org/10.1 038/s41598-019-43886-0

Cao Y, Wu SH, Dai YC (2012) Species clarification of the prize medicinal Ganoderma mushroom "Lingzhi". Fungal Diversity 56(1):49-62. https://doi. org/10.1007/s13225-012-0178-5

Cheng T, Chepkirui C, Decock C, Matasyoh J, Stadler M (2019) Sesquiterpenes from an eastern African medicinal mushroom belonging to the genus Sanghuangporus. Journal of Natural Products 82(5):1283-1291. https://doi. org/10.1021/acs.jnatprod.8b01086

Chepkirui C, Cheng T, Matasyoh J, Decock C, Stadler M (2018) An unprecedented spiro [Furan-2,1'-indene]-3-one derivative and other nematicidal and antimicrobial metabolites from Sanghuangporus sp. (Hymenochaetaceae, Basidiomycota) collected in Kenya. Phytochemistry Letters 25:141-146. https://doi.org/10.1016/j.phytol.2018.04.022

Dai YC (2010) Hymenochaetaceae in China. Fungal Diversity 45(1):131-343. https://doi.org/10.1007/s13225-010-0066-9

Dai YC, Zhou LW, Hattori T, Cao Y, Stalpers JA, Ryvarden L, Buchanan P, Oberwinkler F, Hallenberg N, Liu PG, Wu SH (2017) Ganoderma lingzhi (Polyporales, Basidiomycota): the scientific binomial for the widely cultivated medicinal fungus Lingzhi. Mycological Progress 16(11-12):1051-1055. https:// doi.org/10.1007/s11557-017-1347-4

Edler D, Klein J, Antonelli A, Silvestro D (2021) raxmIGUI 2.0: a graphical interface and toolkit for phylogenetic analyses using RAxML. Methods in Ecology and Evolution 12(2):373-377. https://doi.org/10.1111/2041-210x.13512

Gardes M, Bruns TD (1993) ITS primers with enhanced specifity for Basidiomycetes: application to identification of mycorrhizae and rusts. Molecular Ecology 2(2):113-118. https://doi.org/10.1111/j.1365-294x.1993. tb00005.x
Ghobad-Nejhad M (2015) Collections on Lonicera in Northwest Iran represent an undescribed species in the Inonotus linteus complex (Hymenochaetales). Mycological Progress 14(10):90. https://doi.org/10.1007/s11557-015-1100-9

Guindon S, Gascuel O (2003) A simple, fast and accurate method to estimate large phylogenies by maximum-likelihood. Systematic Biology 52(5):696-704. https://doi.org/10.1080/10635150390235520

Han JG, Hyun MW, Kim CS, Jo JW, Cho JH, Lee KH, Kong WS, Han SK, Oh J, Sung GH (2016) Species identity of Phellinus linteus (sanghuang) extensively used as a medicinal mushroom in Korea. Journal of Microbiology 54(4):290-295. https://doi.org/10.1007/s12275-016-5520-2

He Z, Luo T, Fan F, Zhang P, Chen Z (2019a) Universal identification of lethal amanitas by using Hyperbranched rolling circle amplification based on $a$ amanitin gene sequences. Food Chemistry 298:125031. https://doi.org/10.101 6/j.foodchem.2019.125031

He Z, Su Y, Li S, Long P, Zhang P, Chen Z (2019b) Development and evaluation of isothermal amplification methods for rapid detection of lethal Amanita species. Frontiers in Microbiology 10:1523. https://doi. org/10.3389/fmicb.2019.01523

Hofstetter V, Buyck B, Eyssartier G, Schnee S, Gindro K (2019) The unbearable lightness of sequenced-based identification. Fungal Diversity 96(1):243-284. https://doi.org/10.1007/s13225-019-00428-3

Huo J, Zhong S, Du X, Cao Y, Wang W, Sun Y et al (2020) Whole-genome sequence of Phellinus gilvus (mulberry Sanghuang) reveals its unique medicinal values. Journal of Advanced Research 24:325-335. https://doi.org/1 0.1016/j.jare.2020.04.011

Kapli P, Lutteropp S, Zhang J, Kobert K, Pavlidis P, Stamatakis A, Flouri T (2017) Multi-rate Poisson tree processes for single-locus species delimitation under maximum likelihood and Markov chain Monte Carlo. Bioinformatics 33:16301638. https://doi.org/10.1093/bioinformatics/btx025

Katoh K, Kuma K, Toh H, Miyata T (2005) MAFFT version 5: improvement in accuracy of multiple sequence alignment. Nucleic Acids Research 33(2):511518. https://doi.org/10.1093/nar/gki198

Katoh K, Standley DM (2013) MAFFT multiple sequence alignment software version 7: improvements in performance and usability. Molecular Biology and Evolution 30(4):772-780. https://doi.org/10.1093/molbev/mst010

Kumar S, Stecher G, Li M, Knyaz C, Tamura K (2018) MEGA X: molecular evolutionary genetics analysis across computing platforms. Molecular Biology and Evolution 35(6):1547-1549. https://doi.org/10.1093/molbev/msy096

Lin WC, Deng JS, Huang SS, Wu SH, Lin HY, Huang GJ (2017) Evaluation of antioxidant, anti-inflammatory and anti-proliferative activities of ethanol extracts from different varieties of Sanghuang species. RSC Advances 7(13): 7780-7788. https://doi.org/10.1039/c6ra27198g

Lizardi PM, Huang X, Zhu Z, Bray-Ward P, Thomas DC, Ward DC (1998) Mutation detection and single-molecule counting using isothermal rolling-circle amplification. Nature Genetics 19(3):225-232. https://doi.org/10.1038/898

Nilsson M, Krejci K, Koch J, Kwiatkowski M, Gustavsson P, Landegren U (1997) Padlock probes reveal single-nucleotide differences, parent of origin and in situ distribution of centromeric sequences in human chromosomes 13 and 21. Nature Genetics 16(3):252-255. https://doi.org/10.1038/ng0797-252

Nilsson M, Malmgren H, Samiotaki M, Kwiatkowski M, Chowdhary BP, Landegren U (1994) Padlock probes: circularizing oligonucleotides for localized DNA detection. Science 265(5181):2085-2088. https://doi.org/10.1126/science. 7522346

Nilsson RH, Larsson K-H, Taylor AFS, Bengtsson-Palme J, Jeppesen TS, Schigel D, Kennedy P, Picard K, Glöckner FO, Tedersoo L, Saar I, Kõljalg U, Abarenkov K (2019) The UNITE database for molecular identification of fungi: handling dark taxa and parallel taxonomic classifications. Nucleic Acids Research 47(D1):D259-D264. https://doi.org/10.1093/nar/gky1022

Nilsson RH, Ryberg M, Kristiansson E, Abarenkov K, Larsson K-H, Kõljalg U (2006) Taxonomic reliability of DNA sequences in public sequence databases: a fungal perspective. PLoS One 1(1):e59. https://doi.org/10.1371/journal.pone. 0000059

Pattengale ND, Alipour M, Bininda-Emonds ORP, Moret BME, Stamatakis A (2010) How many bootstrap replicates are necessary? Journal of Computational Biology 17(3):337-354. https://doi.org/10.1089/cmb.2009.0179

Posada D (2008) jModelTest: phylogenetic model averaging. Molecular Biology and Evolution 25(7):1253-1256. https://doi.org/10.1093/molbev/msn083

Raja HA, Baker TR, Little JG, Oberlies NH (2017) DNA barcoding for identification of consumer-relevant mushrooms: a partial solution for product certification? Food Chemistry 214:383-392. https://doi.org/10.101 6/j.foodchem.2016.07.052 
Redhead SA, Ginns J (2006) (1738) proposal to conserve the name Poria cocos against Daedalea extensa (Basidiomycota). Taxon 55(4):1027-1028. https://doi. org/10.2307/25065702

Rodrigues AM, Najafzadeh MJ, de Hoog GS, Camargo ZP (2015) Rapid identification of emerging human-pathogenic Sporothrix species with rolling circle amplification. Frontiers in Microbiology 6:1385. https://doi.org/10.3389/ fmicb.2015.01385

Ronquist F, Teslenko M, van der Mark P, Ayres D, Darling A, Höhna S et al (2012) MrBayes 3.2: efficient Bayesian phylogenetic inference and model choice across a large model space. Systematic Biology 61(3):539-542. https://doi. org/10.1093/sysbio/sys029

Schoch CL, Robbertse B, Robert V, Vu D, Cardinali G, Irinyi L et al (2014) Finding needles in haystacks: linking scientific names, reference specimens and molecular data for fungi. Database 2014:bau061. https://doi.org/10.1093/data base/bau061

Shao Y, Guo H, Zhang J, Liu H, Wang K, Zuo S, Xu P, Xia Z, Zhou Q, Zhang H, Wang $X$, Chen A, Wang Y (2020) The genome of the medicinal macrofungus Sanghuang provides insights into the synthesis of diverse secondary metabolites. Frontiers in Microbiology 10:3035. https://doi.org/10.3389/ fmicb.2019.03035

Stamatakis A (2014) RAxML version 8: a tool for phylogenetic analysis and postanalysis of large phylogenies. Bioinformatics 30(9):1312-1313. https://doi. org/10.1093/bioinformatics/btu033

Stecher G, Tamura K, Kumar S (2020) Molecular evolutionary genetics analysis (MEGA) for macOS. Molecular Biology and Evolution 37(4):1237-1239. https:// doi.org/10.1093/molbev/msz312

Tian XM, Yu HY, Zhou LW, Decock C, Vlasák J, Dai YC (2013) Phylogeny and taxonomy of the Inonotus linteus complex. Fungal Diversity 58(1):159-169. https://doi.org/10.1007/s13225-012-0202-9

Tomšovský M (2015) Sanghuangporus pilatii, a new combination, revealed as European relative of Asian medicinal fungi. Phytotaxa 239(1):82-88. https:// doi.org/10.11646/phytotaxa.239.1.8

Trilles L, Wang B, Firacative C, Lazéra MS, Wanke B, Meyer W (2014) Identification of the major molecular types of Cryptococcus neoformans and C. gattii by Hyperbranched rolling circle amplification. PLoS One 9:e94648. https://doi. org/10.1371/journal.pone.0094648

Turland NJ, Wiersema JH, Barrie FR, Greuter W, Hawksworth DL, Herendeen PS et al (2018) International code of nomenclature for algae, fungi, and plants (Shenzhen code) adopted by the nineteenth international botanical congress Shenzhen, China, July 2017. Koeltz Botanical Books, Glashütten

Wang XC, Xi RJ, Li Y, Wang DM, Yao YJ (2012) The species identify of the widely cultivated Ganoderma, 'G. Iucidum' (Ling-zhi), in China. PLoS One 7:e40857. https://doi.org/10.1371/journal.pone.0040857

White TJ, Bruns TD, Lee SB, Taylor JW (1990) Amplification and direct sequencing of fungal ribosomal RNA genes for phylogenetics. In: Innis MA, Gelfand DH, Sninsky JJ, White TJ (eds) PCR protocols: a guide to methods and applications. Academic, San Diego, pp 315-322

Wu F, Zhou LW, Yang ZL, Bau T, Li TH, Dai YC (2019a) Resource diversity of Chinese macrofungi: edible, medicinal and poisonous species. Fungal Diversity 98(1):1-76. https://doi.org/10.1007/s13225-019-00432-7

Wu SH, Chang CC, Wei CL, Jiang GZ, Cui BK (2019b) Sanghuangporus toxicodendri sp. nov. (Hymenochaetales, Basidiomycota) from China. MycoKeys 57:101-111. https://doi.org/10.3897/mycokeys.57.36376

Wu SH, Dai YC, Hattori T, Yu TW, Wang DM, Parmasto E et al (2012a) Species clarification for the medicinally valuable 'sanghuang' mushroom. Botanical Studies 53:135-149

Wu SH, Kirk PM, Redhead SA, Stalpers JA, Dai YC, Norvell LL, Yang ZL, Ryvarden L, Su CH, Li Y, Zhuang WY, Yao YJ, Chen CJ, Chen LC, Yu ZH, Wang XC (2012b) Resolution of the nomenclature for niu-chang-chih (Taiwanofungus camphoratus), an important medicinal polypore. Taxon 61(6):1305-1310. https://doi.org/10.1002/tax.616011

Wu SH, Yao YJ, Wang XC, Kirk PM, Redhead SA, Stalpers JA, Dai YC, Norvell LL, Yang ZL, Ryvarden L, Su CH, Li Y, Zhuang WY, Chen CJ, Chen LC, Yu ZH (2012c) (2101) proposal to conserve the name Ganoderma camphoratum (Taiwanofungus camphoratus) (Polyporales) with a conserved type. Taxon 61(6):1321-1322. https://doi.org/10.1002/tax.616015

Yao YJ, Li Y, Du Z, Wang K, Wang XC, Kirk PM et al (2020) On the typification of Ganoderma sichuanense (Agaricomycetes)-the widely cultivated Lingzhi medicinal mushroom. International Journal of Medicinal Mushrooms 22(1): 45-54. https://doi.org/10.1615/IntJMedMushrooms.2019033189
Yao YJ, Wang XC, Wang B (2013) Epitypification of Ganoderma sichuanense J.D. Zhao \& X.Q. Zhang (Ganodermataceae). Taxon 62(5):1025-1031. https://doi. org/10.12705/625.10

Zhou LW (2020) Systematics is crucial for the traditional Chinese medicinal studies and industry of macrofungi. Fungal Biology Reviews 34(1):10-12. https://doi.org/10.1016/j.fbr.2019.10.002

Zhou LW, Ghobad-Nejhad M, Tian XM, Wang YF, Wu F (2020) Current status of 'Sanghuang' as a group of medicinal mushrooms and their perspective in industry development. Food Reviews International:1-19. https://doi.org/10.1 080/87559129.2020.1740245

Zhou LW, Qin WM (2012) Inonotus tenuicontextus sp. nov. (Hymenochaetaceae) from Guizhou, Southwest China with a preliminary discussion on the phylogeny of its kin. Mycological Progress 11(3):791-798. https://doi.org/10.1 007/s11557-011-0792-8

Zhou LW, Vlasák J, Decock C, Assefa A, Stenlid J, Abate D, Wu SH, Dai YC (2016) Global diversity and taxonomy of the Inonotus linteus complex (Hymenochaetales, Basidiomycota): Sanghuangporus gen. nov., Tropicoporus excentrodendri and T. guanacastensis gen. et spp. nov., and 17 new combinations. Fungal Diversity 77(1):335-347. https://doi.org/10.1007/s13225015-0335-8

Zhou X, Kong F, Sorrell TC, Wang H, Duan Y, Chen SC (2008) Practical method for detection and identification of Candida, Aspergillus, and Scedosporium spp. by use of rolling-circle amplification. Journal of Clinical Microbiology 46(7):24232427. https://doi.org/10.1128/JCM.00420-08

Zhu L, Song J, Zhou JL, Si J, Cui BK (2019) Species diversity, phylogeny, divergence time, and biogeography of the genus Sanghuangporus (Basidiomycota). Frontiers in Microbiology 10:812. https://doi.org/10.3389/ fmicb.2019.00812

Zhu L, Xing JH, Cui BK (2017) Morphological characters and phylogenetic analysis reveal a new species of Sanghuangporus from China. Phytotaxa 311(3):270276. https://doi.org/10.11646/phytotaxa.311.3.7

\section{Publisher's Note}

Springer Nature remains neutral with regard to jurisdictional claims in published maps and institutional affiliations.

Ready to submit your research? Choose BMC and benefit from:

- fast, convenient online submission

- thorough peer review by experienced researchers in your field

- rapid publication on acceptance

- support for research data, including large and complex data types

- gold Open Access which fosters wider collaboration and increased citations

- maximum visibility for your research: over $100 \mathrm{M}$ website views per year

At BMC, research is always in progress.

Learn more biomedcentral.com/submissions 